\title{
Struktur? Physis? Situation? Zur Erklärung von Gewalt
}

\author{
Thomas Kron · Lena M. Verneuer
}

Angenommen: 10. Dezember 2020 / Online publiziert: 23. Dezember 2020

(C) Der/die Autor(en) 2020

Zusammenfassung Die soziologische Gewaltforschung verharrt vor allem in der begrifflichen Auseinandersetzung um das Gewaltverständnis. Der vorliegende Beitrag lenkt den Blick dagegen auf die Erklärung von Gewalt und zieht das strukturindividualistische Grundmodell soziologischer Erklärung von Coleman und Esser heran, um die Diskussionslinien zu vereinen. Hierfür wird skizziert, welche Diskussionsstränge in der Gewaltforschung auszumachen sind und welche Leerstellen nach wie vor bestehen. Der Bezugsrahmen des Grundmodells wird daran anschließend unter Rückgriff auf Randall Collins' situationale Perspektive auf Gewalt entlang der Logik der Situation, der Selektion und der Aggregation vertiefend vorgestellt. Es wird gezeigt, dass dieser Bezug ermöglicht, die scheinbar unvereinbaren Positionen innerhalb der Gewaltforschung nicht nur zu verorten, sondern explanatorisch zu integrieren.

Schlüsselwörter Gewalt · Erklärung · Grundmodell der soziologischen Erklärung · Randall Collins

\footnotetext{
T. Kron $(\varangle) \cdot$ L. M. Verneuer

Institut für Soziologie, RWTH Aachen, Eilfschonsteinstr. 7, 52062 Aachen, Deutschland

E-Mail: tkron@soziologie.rwth-aachen.de

L. M. Verneuer

E-Mail: 1verneuer@soziologie.rwth-aachen.de
} 


\section{Structure? Physis? Situation? On the explanation of violence}

Abstract Sociological research on violence keeps circling around conceptual questions over the understanding of violence. In contrast, the present contribution turns to the explanation of violence and draws on Coleman's and Esser's structural-individualistic model of sociological explanation to bring together the different lines of discussion. To this end, the article sketches out the strands of discussion that can be identified in research on violence as well as the gaps that still exist. Drawing on Randall Collins' situational perspective on violence, Esser's model is then presented in greater depth with reference to the logic of situation, selection and aggregation. It is shown that this reference makes it possible not only to situate the seemingly irreconcilable positions within the study of violence, but also to integrate them into a single explanatory framework.

Keywords Violence $\cdot$ Explanation $\cdot$ Model of sociological explanation $\cdot$ Randall Collins

\section{Structure ? Physis ? Situation? Sur l'explication de la violence}

Résumé La sociologie de la violence s'attache avant tout à débattre de la notion de violence. Le présent article attire l'attention sur l'explication de la violence et fait appel au modèle de base d'explication sociologique de l'individualiste structurel de Colman et Esser pour unifier les différentes lignes de discussion. Pour ce faire, cet article esquisse les axes de discussion identifiables dans la sociologie de la violence ainsi que ses lacunes persistantes. Le cadre de référence du modèle de base est ensuite présenté de manière approfondie en recourant à la perspective situationnelle sur la violence de Randall Collins suivant les logiques de situation, de sélection et d'agrégation. Il est montré que cette référence permet non seulement de situer les positions apparemment incompatibles au sein de la sociologie de la violence mais aussi de les intégrer dans une visée explicative.

Mots-clés Violence · Explication · Modèle de base de l'explication sociologique · Randall Collins

Die Tatsache, dass gebildete Intellektuelle glauben können, dass das, was sie symbolische Gewalt nennen, irgendetwas mit physischer Gewalt zu tun habe, zeigt, wie ungewohnt es für die meisten Intellektuellen ist, in mikrosoziologischen Begriffen zu denken, und wie wenig wir mit realer Gewalt vertraut sind.

Randall Collins (2011, S. 42)

\section{Der soziologische Diskurs um Gewalt}

Das aktuelle Interesse der soziologischen Gewaltforschung (siehe etwa Braun und Kron 2020; Equit et al. 2016; Hartmann 2013; Hoebel und Knöbl 2019; Hoebel und Malthaner 2019; Paul und Schwalb 2015; Staudigl 2014) gleicht einer zykli- 
schen Wiederholung und Aufrechterhaltung derjenigen Argumente, wie sie die sogenannten „Mainstreamer“ und „Innovateure“ ( siehe zur Debatte Hüttermann 2004; Imbusch 2004; Nedelmann 1997; von Trotha 1997) bereits seit den 1990er-Jahren austauschen. Die damals vorgebrachten und heute stets mitgeführten Argumente der sogenannten ,,neuen Gewaltsoziologie“ gegenüber der damals prominenten Gewaltursachenforschung beziehen sich - unter anderem - auf die theoretische wie empirische Konzentration auf die Ursachen und Motive von Gewalt: Diese als ,körperlose[] Faktorenkombinatorik“ (Sutterlüty 2015, S. 249) oder als „,blutleere[] Gewaltanalyse" (Nedelmann 1997, S. 62) bezeichneten Forschungsarbeiten wurden als unzureichend für eine soziologische Gewaltforschung erachtet, insbesondere da sie den eigentlichen Gegenstand - Gewalt - durch die Fokussierung auf die zeitlich vorgelagerten Ursachen ausblenden würden (z.B. Nedelmann 1997; von Trotha 1997). Damit einher ging die Auseinandersetzung mit dem Gewaltbegriff, dessen inflationäre Ausweitung auf alles, was potenziell geeignet ist, die Freiheit des Menschen einzuschränken, nicht im Sinne der neuen Gewaltsoziologie war (und ist). ${ }^{1}$ Favorisiert wurde und wird daher von ihren Vertreter:innen ein enger Gewaltbegriff, der sich der Definition von Heinrich Popitz (1992) anschließt und die Physis, das Verletzen und Erleiden, in den Mittelpunkt stellt. ${ }^{2}$

Die von Nedelmann (1997) als „Scheideweg“ markierte Abgrenzung zu den sogenannten „Mainstreamern“ wurde in unterschiedlicher Form aufgenommen und kritisch diskutiert - eine Debatte, die ob ihrer Kategorien-Unschärfe sowie der Zielrichtung als „,kurios“ bezeichnet wurde (Imbusch 2004). Neben der Frage, ob und wen man welcher Gruppe überhaupt zuordnen kann und wieviel Gehalt ebendiese „Gegnerschaft“ (Hüttermann 2004, S. 108) mit sich bringt, bezieht sich ein fortlaufend diskutierter Aspekt auf die Auseinandersetzung mit der begrifflichen Engführung von Gewalt durch die „Innovateure“, die wiederum kritisch u.a. als „Radikalisierung der normativen Aufladung des Begriffs durch seine Einengung auf das Erleiden extremer physischer Gewalt“ (Nunner-Winkler 2004, S. 26) bezeichnet wurde und manchen Kritiker:innen der ,neue Gewaltforschung“ wiederum als zu restriktiv im Angesicht möglicher „Erklärungsbegehren“ gilt (Reemtsma 2017). Im Geiste einer Erweiterung der Perspektive hat Peter Imbusch (2017) ein Plädoyer für die Nützlichkeit der Berücksichtigung von Phänomenen „struktureller Gewalt““ vorgelegt und den von Johan Galtung (1975) eingeführten Begriff sowohl kritisch begutachtet als auch für die aktuelle Debatte zu rehabilitieren versucht. Die mit die-

\footnotetext{
1 Kritisiert wird von den „Innovateuren“, dass ein weiter Begriff von Gewalt diesen konsequentialistisch ausdeutet und mit jeglicher Schädigung gleichsetzt. Sofern alles irgendjemanden schädigen kann, kann nach dieser Vorstellung auch alles ,strukturelle Gewalt“ werden - z.B. auch moderne Intimsysteme mit ihrer Formvorschrift des Liebens (Fuchs 1999), welche bei Erwartungsenttäuschungen massive psychischseelische Schädigungen hervorrufen können, die sogar in Intimizid (Marneros 2008) und (Selbst-)Tötung enden können (Lester et al. 2004). Ähnliches lässt sich in Bezug auf politische Institutionen sagen, die Freiheiten nicht nur gewähren, sondern auch beschränken und selbst einen physisch gewaltförmigen Charakter aufweisen können: „Alle politischen Gebilde sind Gewaltgebilde“, heißt es schon bei Weber (1980, S. 520). Letztlich wird damit die Gesellschaft mit Gewaltverhältnissen gleichgesetzt.

2 Popitz (1992, S. 48) definiert Gewalt als ,eine Machtaktion, die zur absichtlichen körperlichen Verletzung anderer führt, gleichgültig, ob sie für den Agierenden ihren Sinn im Vollzug selbst hat (als bloße Aktionsmacht) oder, in Drohungen umgesetzt, zu einer dauerhaften Unterwerfung (als bindende Aktionsmacht) führen soll“".
} 
sem Begriff arbeitende Forschung lege zentrale Gewaltphänomene der Gegenwart inklusive ihrer Konstitutionsbedingungen frei, wie etwa die neoliberal verursachte „Multiexklusion“, und nähme ,,auch solche Schädigungen in den Blick [...], die jenseits durch konkrete Täter verübter Gewaltaktionen liegen und sich quasi unsichtbar aus den Sozialstrukturen und der sozioökonomischen Verfasstheit einer Gesellschaft ergeben“ (Imbusch 2017, S. 38). Gerade angesichts der zunehmenden unsichtbaren Verursachungen von Schädigungen sei der Begriff der „strukturellen Gewalt“ von ,,besonderer Bedeutung“, da er zur ,Skandalisierung gesellschaftlicher Umstände" geeignet sei, welche ansonsten übersehen würden. Auf diese Weise werde die durch ,moralische Anprangerung“ notwendige Aufmerksamkeit erzeugt und politische Problemlösungen angemahnt (ebd.). Imbuschs Argumentationslinie bezüglich eines erweiterten Gewaltbegriffes schließt damit an die Kritik von Markus Schroer an einem engen Gewaltverständnis an: Schroer argumentiert, dass „die Gewaltsoziologie zentrale Gewaltphänomene der Gegenwartsgesellschaft nicht in den Blick bekommt, wenn sie sich einseitig auf den Begriff der physischen Gewalt kapriziert" (Schroer 2004, S. 155). Er sieht in der Verengung des Gewaltbegriffs durch die „Innovateure“ ein Signum der zeitgenössischen Moderne, die nur das wahrnehme, was visuell erfassbar sei. Der Begriff der ,strukturellen Gewalt“ sei für die Analyse „unsichtbarer“ Formen von Gewalt unverzichtbar. ${ }^{3}$ Bei diesen könne die Gewaltanwendung nämlich nicht auf individuelle Personen zurückgeführt werden, z.B. bei Prozessen der Individualisierung und Globalisierung und der daraus folgenden sozialen Ungleichheit, welche Leid in großen Bevölkerungsteilen zur Folge habe. In den Gewaltanalysen der „Innovateure“ - z.B. in jenen dichten Beschreibungen von direkter Gewalt, wie sie etwa Wolfgang Sofsky (1996) vorgelegt hat - sieht Schroer vor allem eine Strukturvergessenheit: So versuche Sofsky nicht einmal, ,eine Anbindung an gesellschaftsstrukturelle Gegebenheiten herzustellen, wodurch die Ausbrüche der Gewalt [...] zu zeitlosen, kontextfreien Geschehnissen stilisiert würden“, das Phänomen Gewalt damit „,gewissermaßen für sich selbst“ stehe, und die Rekonstruktion „durch keinerlei Erklärungen getrübt“ werden soll (Schroer 2004, S. $161 \mathrm{f}$.).

Kritisiert wird also von Imbusch, Schroer und anderen, dass das enge physische Gewaltverständnis, methodisch flankiert von ebenjenen dichten Beschreibungen, nicht kontextualisiert und in kein Erklärungsmodell eingepasst wird, welches strukturelle Randbedingungen einbezieht. Je mehr jegliche „Ursachenerklärung“ diskreditiert und dichte Beschreibungen der Gewaltphänomene als Königsweg der Gewaltforschung verkauft werde, desto weniger angemessen seien die Analysen den zeitgenössischen Gewaltformen (ebd., S. 162f.): „Was die dichten Beschreibungen

\footnotetext{
3 Hartmann und Hoebel (2020, S. 74) greifen die Kritik am vorgeblich ,kaum reflektierten ,Anwesenheitsbias “" der neuen Gewaltforschung auf - zu der sie auch Randall Collins 'situationistische Analysen zählen -, um ihr vorzuwerfen, bestimmte soziale Phänomene durch deren Verschleierung mitzutragen. Die „,[v]isuelle[n] Verengungen“ seien zwar ,,heuristisch produktiv“, aber „zugleich ein zentraler Grund dafür, dass sich kollektive Aufmerksamkeitspräferenzen ausbilden, die eher politischen und medialen Logiken folgen denn (sozial-)wissenschaftlichen“. Das impliziere Bewertungen derart, ,dass bestimmte Gewaltformen gesellschaftlich wichtiger seien als andere. Sie tragen womöglich, wenn auch unabsichtlich, dazu bei, dass diejenigen Akteur_innen, denen ohnehin schon keine nennenswerte Stimme im gesellschaftlichen Diskurs über das Antun und Erleiden von Gewalt zukommt, auch über die Forschung wenig Gehör finden.“
} 
der Gewalt selbst nicht zu erfassen vermögen, sind die für moderne Gesellschaften gerade typischen Gewaltformen, die sich verstärkt zwischen Abwesenden abspielen. Darüber hinaus richtet sich ihr Augenmerk auf die Eruptionen der Gewalt, auf die Situationen, in denen es - für alle Augen sichtbar - zu Gewalttaten kommt. Blind aber sind sie für die sich in Institutionen, Organisationen und Funktionssystemen ablagernden Formen der Gewalt, die zwar weniger offensichtlich, deshalb aber nicht weniger nachhaltig Lebenschancen beeinträchtigen und Leben zerstören können.“ (ebd., S. 164) Es ist leicht zu sehen, dass eine solche begriffliche Erweiterung des Gewaltverständnisses immer auch eine zunehmende semantische Unschärfe mit sich bringt ${ }^{4}$, welche dann wieder im Sinne der ,Innovateure“ kritisiert werden kann. So muss Schroer durch seine Ausweitung des Gewaltbegriffs in Kauf nehmen, dass er u.a. die Unterscheidung von Gewalt und Zwang oder Gewalt und Leid unterläuft. Eine solche Vorstellung von Gewalt erweitert ihren Bezugsrahmen so weit, dass der Vorwurf einer Inflationierung des Gewaltbegriffs wieder möglich wird.

Wie in gebotener Kürze skizziert, dreht sich ein Teil der aktuellen Diskussionen innerhalb der deutschen soziologischen Gewaltforschung also darum, den Gewaltbegriff definitorisch ein- bzw. abzugrenzen (Nunner-Winkler 2004). Vordergründig geht es in diesen Diskursen darum herauszufinden, welche Ingredienzien der Gewaltbegriff beinhalten sollte. Die soziologische Gewaltforschung befindet sich so gesehen nach wie vor - so resümieren es Hoebel und Malthaner (2019, S. 7) - ,in einer Phase des Probierens, Kritisierens und Sortierens" -, eine fruchtbare Fortentwicklung ist allerdings nicht zu erkennen. Anders formuliert, ,eine kuriose Debatte wiederholt sich“ (Koepp und Schattka 2020; siehe zusammenfassend Braun 2020). Nach wie vor lässt sich eine Diskussion um die Verengung und Erweiterung des Gewaltbegriffes beobachten, die in aktuellen Veröffentlichungen als ,,analytisch unfruchtbare Begriffsoszillation“ (Hoebel und Knöbl 2019, S. 29) oder als ,zirkulär“ (Hoebel und Koloma Beck 2017, S. 2) charakterisiert wird. Diese Oszillation um einen vermuteten Bedeutungskern wird von den Protagonisten des Diskurses durchaus selbst gesehen, etwa wenn Imbusch (2017, S. 38) dem Begriff der ,strukturellen Gewalt" die Bedeutung für die Gewaltforschung zuspricht, ,gleichsam in Umkehrung des Vorwurfs einer Überdehnung des Gewaltbegriffs [...] eine nicht zu rechtfertigende Engführung des Gewaltverständnisses“ auf physische Gewalt zu vermeiden. Derartige Diskussionen um eine eher mikro- oder makroskopische Betrachtung von Gewaltphänomenen führen ,regelmäßig in unfruchtbare Begriffsdebatten“, so Hartmann und Hoebel (2020, S. 67), ,die dem Erkenntnisstand über Gewaltphänomene in der Regel kaum etwas hinzufügen“.

Der soziologische Gewaltforschungsdiskurs mag sich einig sein über den unfruchtbaren Zustand der begrifflichen Klärungsversuche. Die mittlerweile zwei Jahr-

\footnotetext{
4 Diese semantische Unschärfe bedeutet nicht, dass man von einer ,grundsätzlichen Unbestimmbarkeit“ (Imbusch 2017, S. 35) ausgehen muss, sondern zielt darauf, den Begriff der Gewalt ,auf einem Kontinuum zu verorten, das von Gewaltfreiheit über Gewaltrelevanz bis hin zu Gewalttätigkeit verläuft“ (Unterkofler 2016, S. 134; siehe Kron 2020a, 2020b, S. 307 ff. zur Modellierung der Unschärfe von ,emotionaler Energie" in Gewaltsituationen sowie zur begrifflichen Abgrenzung von Gewalt zu anderen Angriffen). Alle Faktoren von Gewalt, beispielsweise die Verletzbarkeit (siehe Nungesser 2019), scheinen über Zugehörigkeitsgrade zu verlaufen, sodass Gewalt den Phänomenen hybrider Sozialität (Kron 2015) zugerechnet werden kann.
} 
zehnte andauernden Auseinandersetzungen haben gezeigt, dass das Unterfangen der Begriffsklärung allein für sich nicht viel Hoffnung auf einen Erkenntnisgewinn macht, denn im Kampf um die ,richtige“ begriffliche Fassung von Gewalt kommt man höchstens darauf, dass zur gewünschten Beseitigung semantischer Differenzen weitere Begriffe fehlen (Endreß 2014, S. 100f.) - und für diese Begriffe würden vermutlich, wenn sie doch gefunden würden, wiederum semantische Unklarheiten auftauchen und derart für sie dasselbe gelten.

Zugleich ist der Gegenstandsbezug nach wie vor ungeklärt. Es gibt keine Einigung darüber, welche sozialen Phänomene mit dem Begriff „Gewalt“ adressiert werden sollen: Ist Gewalt auf die Verletzung der Physis zu begrenzen oder können bzw. sollten oder müssen strukturelle und kulturelle Einschränkungen von Handlungsfreiheiten in den Gewaltbegriff integriert werden? Sind normative Elemente konstitutiver Bestandteil des Begriffs? Sind die Intentionen vonseiten des Täters konstitutiv oder muss der Sinngehalt jeder Gewalt in der wechselseitigen Erzeugung durch Täter und Opfer gesehen werden? Geht es bei Gewalt im Kern um Verletzungen durch direkte körperliche Angriffe und Übergriffe oder auch um (irgendwie geartete) Verletzungen als Folge auch nicht-körperlicher Einwirkungen? Sind Verletzungen das indikatorische Merkmal von Gewalt oder das damit verbundene oder auch anders entstandene Leid? Geht es um das Erleiden von Schmerzen? Ist ausschließlich der Körper von Gewalt betroffen oder der Leib? Sind Gewaltsituationen analytisch hinreichend als dyadische Situationen begriffen oder muss „,der Dritte“ notwendig einbezogen werden?

Diese Fragen, die nicht mal annähernd das ganze Spektrum an diskutierten Unterscheidungs- und Definitionsfragen wiedergeben, zeigen: Die Klärung des Gegenstandsbezugs ohne eine klare Kopplung an eine Erklärungsdimension ist blind und damit unendlich in dem Bemühen, mehr zu sehen. Die Klärung des Gegenstandsbezugs muss, so unsere These, begleitet werden durch die Erörterung einer angemessenen Erklärungsheuristik. In dem Diskurs der soziologischen Gewaltforschung wird stattdessen - wie gezeigt - um den Gewaltbegriff in Bezug auf spezifische Gegenstände gerungen in der Hoffnung, dadurch uno actu die Erklärungsleistung zu kondensieren (dazu auch Hoebel und Knöbl 2019, S. 24ff.). „Strukturelle Gewalt““ erkläre folglich jene Gewalt, die sich als Reduktion von Lebenschancen darstelle, was eben ein enger Gewaltbegriff nicht leiste. Schroer etwa ist zwar um die Betrachtung des Zusammenspieles beider Gewaltzugänge - via Physis und Struktur bemüht, unterlässt es allerdings, diese Verbindung anhand möglicher Erklärungsmodelle zu suchen, und bleibt stattdessen bei dem Versuch der Entwicklung eines integralen Gewaltbegriffs stehen - den es aber, so Schroer (2004, S. 165) selbst, nicht geben könne. Der Eindruck entsteht, dass Fragen nach der Erklärung von Gewalt und nach der angemessenen Begrifflichkeit bis zur Unkenntlichkeit ineinander verwoben werden: Wer wie die „Innovateure“ den Schlüssel zur Gewalterklärung in der Gewalthandlung selbst und damit mittels eines engen Gewaltbegriffs zu finden sucht und sich auf dichte Beschreibungen zur Rekonstruktion von Gewalt fokussiert, wird sich gleichzeitig primär auf die Wie-Frage konzentrieren. Zugleich haben große Teile der Soziologie und auch der Gewaltforschung - implizit oder explizit ein Interesse an der Warum-Frage. In dem Diskurs der Gewaltforschung entscheidet einerseits die meist unreflektiert mitlaufende Frage zur Erklärungsheuristik darüber, 
was in der Gegenstandsdimension als Gewalt in den Blick kommt. Und vice versa: Was als Gewalt definiert wird, hegt andererseits das ein, was als Erklärungsfaktoren für die mit diesem Begriff verbundenen Phänomene in Betracht gezogen wird.

Insgesamt zeichnet sich dieser Gewaltforschungsdiskurs durch eine weitgehende Erklärungsvergessenheit aus. Es ist bis in die jüngste Gegenwart praktisch unterlassen worden, die Frage nach der Erklärung von Gewalt analytisch separat zu behandeln, um in diesem Zuge die irreführende Diskussion um einen geeigneten Gewaltbegriff aus ihrer Zirkularität zu führen. Dies ist durchaus kurios, wie bereits Imbusch deutlich gemacht hat, denn die ,grundsätzliche Überlegenheit einer Perspektive, die die ,Was'- und ,Wie'-Frage stellt, gegenüber den sich aus der ,Warum'-Frage ergebenden Einsichten scheint nun ebensowenig geklärt zu sein, wie die exklusive Entgegensetzung unschlüssig ist, da man beide Aspekte durchaus unter Komplementaritätsgesichtspunkten betrachten kann“ (Imbusch 2004, S. 130). Konkrete Hinweise (siehe etwa von Trotha 1997, S. $21 \mathrm{f}$.), wie man diese Komplementarität zustande bringt, sind selten (vgl. Hüttermann 2004; Knöbl 2017; Nedelmann 1997). Es bleibt in der Regel bei Hinweisen, dass jene Theorieansätze, die sich eher den Wie-Fragen verpflichtet sehen, durchaus auch Erklärungskraft innehaben. Endreß (2004) bemüht sich in diesem Sinne um das Aufzeigen der Nützlichkeit eines phänomenologischen Ansatzes für die Gewaltforschung - was dann für den Gewaltbegriff bedeutet, der subjektiven Erfahrung der Gewalt einen hohen Stellenwert einzuräumen, was wiederum zumindest auf die Notwendigkeit der Berücksichtigung von Leiblichkeit und eines Dritten hinweise (Staudigl 2014, 2015; vgl. Koloma Beck 2017, S. 66 f.; Lindemann 2015, S. $504 \mathrm{ff}$.) und sich somit von dem engen Gewaltbegriff der ,Innovateure“ verabschiedet, zugleich aber die grundlegende Kritik am engen Gewaltbegriff nicht beseitigt. Knöbl meint, man könne zunächst phänomenologisch beginnen, gewinne dann aber mit der ethnografischen Fortsetzung ,ein schärferes Bild und damit letztlich auch eine bessere Erklärung als lediglich der scheinbare illusionslose Blick auf die nackte Gewalt“ (Knöbl 2017, S. 11). Erklärungen in der Ethnografie zielten zwar nicht auf Aussagen, die die Gültigkeit von Kausalgesetzen für sich in Anspruch nehmen würden, aber: „Wenden wir diesen Befund nun auf die Debatte innerhalb der Gewaltforschung an, so können wir durchaus sagen, dass ethnografische Beschreibungen von Gewalt sehr wohl in der Lage sind, Erklärungsleistungen zu erbringen, ohne auf klare Kausalitäten - und schon gar nicht auf Kausalität durch Motive! - zurückgreifen zu müssen." (ebd., S. 9)

An dieser exemplarischen Beschreibung einer anderen, nämlich ethnografischen Erklärungsleistung wird deutlich: Die Ableitung von Gewalt als Gegenstand aus der Theorie heraus ist für die Erklärung von Gewalt wenig instruktiv, sofern die verwendete Theorie überhaupt einen Erklärungsanspruch hat: Die Theorie wählt denjenigen Gewaltbegriff, welcher die ihr zumutbare Erklärungsleistung gewährleistet. Weder der Kampf um die ,richtige“ Begrifflichkeit von Gewalt, noch die einseitige Orientierung an einer bestimmten Theorie und deren Gewaltzugang (was immer dann Gewalt in der verwendeten Theorie bedeutet) hilft bei der Erklärung von Gewalt wirklich weiter. Ein Grund für die verfahrene Lage könnte darin liegen, dass der Begriff der „Erklärung“ unglücklicherweise ebenso von „fuzzyness“ (Reemtsma 2017, S. 81) geprägt ist wie der Begriff der Gewalt (vgl. Endreß 2014, 
S. 91). Was benötigt wird, ist zunächst eine Vorstellung darüber, wie erklärt werden soll, um sodann mögliche Theorien für ein solches Erklärungsmodell anzubringen, welches dann wiederum einen entsprechenden Gewaltbegriff mitführt.

Das von Thomas Hoebel und Wolfgang Knöbl (2019) veröffentlichte Buch Gewalt erklären! ist angetreten, diese Lücke zu schließen, bietet aber nur bedingt Lösungsansätze. Das Werk beinhaltet eine Nachzeichnung der Begriffsdebatten innerhalb der soziologischen Gewaltforschung inklusive der Schieflagen eben dieser Debatten. Hoebel und Knöbl schlagen zur Systematisierung drei Heuristiken unter den Stichworten Motive, Situationen, Konstellationen als - wenngleich partiell defizitäre - Ordnungskategorien vor, um die jeweils adressierte Erklärungsleistung der aktuellen Ansätze innerhalb der soziologischen Gewaltforschung zu verdeutlichen (ebd., S. 61 ff.). In einer tour de force werden hierbei gängige Ansätze für ungeeignet erklärt. Insbesondere wird angedeutet, dass Collins' Vorschlag einer ,,situationalen Gewaltforschung“, der innerhalb der aufgeführten Diskussion eher der „,neuen Gewaltforschung“ zugerechnet wird als den „Mainstreamern“" , ,über dem Zenit“" (Hoebel und Malthaner 2019) sei. Zum Ausweg erklären Hoebel und Knöbl den eigenen, von Hoebel (2019a, 2019b, 2014) präferierten Ansatz einer auf „Verkettungen und Verstrickungen“ zielenden ,,prozessorientierten Erklärung von Gewalt“. Wir können hier nicht detailliert auf diesen Vorschlag eingehen. Abgesehen von einigen erklärungstheoretischen Unzulänglichkeiten (mangelnde Unterscheidung zwischen notwendigen und hinreichenden Bedingungen, fehlende Integration der Dynamiken von Prozessen in das Modell, fehlende Darlegung handlungstheoretischer Parameter etc.), stimmen wir der Kernaussage von Hoebel und Knöbl zu, dass die Berücksichtigung des Handlungsablaufs für die Erklärung von Gewalt relevant ist. Time matters - allerdings muss deutlich werden, an welcher Stelle der Erklärung die Historie des Geschehens relevant wird und an welcher Stelle nicht, da ansonsten die soziologische Erklärung zur puren Nacherzählung von Ereignissen verkommt.

Wir möchten im Unterschied zum prozesssoziologischen Ansatz in diesem Beitrag den erklärungstheoretischen Bezugsrahmen des sogenannten struktur-individualistischen Grundmodells der soziologischen Erklärung ${ }^{6}$ (Coleman 1991; Esser 1993, S. $91 \mathrm{ff} . ; 1999$ ) heranziehen, um die Diskussionslinien bzgl. des angemessenen Gegenstandsbezugs der Gewaltforschung zu vereinen. Zum einen handelt es sich dabei um ein Erklärungsmodell, das trotz aller bereits dargelegten Kritik daran den Nachweis der Erklärungskraft vielfältig erbracht hat. Man kann dieses Modell zur soziologischen Erklärung diverser sozialer Phänomene heranziehen - dies wird auch von den Kritiker:innen kaum bezweifelt. ${ }^{7}$ Zum anderen hat der Gewaltforschungsdiskurs dieses Modell bislang erstaunlicherweise mehr oder weniger komplett ignoriert ${ }^{8}$,

\footnotetext{
5 Von Letzteren grenzt sich Collins explizit ab (2011, S. $36 \mathrm{ff}$.).

${ }^{6}$ Im Folgenden werden für dieses Modell die Bezeichnungen „Grundmodell der soziologischen Erklärung“ und „Modell der soziologischen Erklärung" synonym verwendet.

7 Diskutabel ist freilich, wo die Grenzen der Erklärungskraft dieses Modells liegen (siehe Kron 2018; Münch 1998).

${ }^{8}$ Siehe exemplarisch jüngst Wolters (2020, S. 120), die die Kausalität, „zumindest in einem deduktivnomologischen Verständnis“, als Gegensatz zur „Interpretation“ sieht, ohne die entsprechenden Vorschläge und Diskussionen im Rahmen des Modells soziologischer Erklärungen etwa über das sogenannte FrameSelection-Modell auch nur zur Kenntnis zu nehmen (grundlegend Esser 2001, 2003b, 2010).
} 
was sich etwa an der von Hartmann und Hoebel referierten These von Hoebel und Knöbl ablesen lässt, jene Ansätze, die sich unter die Stichworte Motive, Situationen und Konstellationen einordnen lassen, würden ,,jeweils einen Aspekt von Gewalt hervorheben - was jedoch zulasten anderer Gesichtspunkte geht" (Hartmann und Hoebel 2020, S. 76). Es ist leicht zu sehen, dass bei Hoebel und Knöbl weiter keine sorgfältige Auseinandersetzung mit diesem Erklärungsmodell erfolgt ist ${ }^{9}$ und deshalb übersehen wurde, dass die gewünschte Integration dort bereits vorliegt inklusive der von ihnen präferierten Prozessorientierung.

Wir schließen dabei an Randall Collins' (2011) Vorschlag an, Gewaltforschung im Sinne einer Analyse von Gewaltsituationen zu betreiben, und zeigen an diesem prominenten Beispiel des aktuellen Gewaltforschungsdiskurses, dass die Klärung der Frage, wie soziologische Erklärungen generell aussehen könnten, hilft, verschiedenen Ansätzen der soziologischen Gewaltforschung einen entsprechenden epistemologischen „Platz“ zuzuordnen.

Collins' situationistischer Ansatz wird, wie gesagt, üblicherweise eher den Vertreter:innen eines engen Gewaltbegriffs zugeschlagen, da Collins sich gegen die Berücksichtigung symbolischer und sozialstruktureller Faktoren zur Erklärung von Gewalt ausspräche. Durch seine mikrosituative Fokussierung auf ,,auslösende Momente der Gewalt“ (Collins und Hartmann 2019, S. 63) klammert er situationsübergreifende Ursachen und Motive jedoch vor allem methodologisch aus. $\mathrm{Zu}$ erkennen ist allerdings zum einen - und deshalb gehen wir von diesem Ansatz aus -, dass Collins seine Kritik an ätiologischen und multifaktoriellen Ansätzen erklärungstheoretisch und nicht begrifflich begründet. Zum anderen expliziert Collins zwar kein elaboriertes Erklärungsmodell, verortet sich aber selbst - teils explizit, teils implizit - im Makro-Mikro-Makro-Gefüge des Grundmodells soziologischer Erklärung (auch wenn Collins als Handlungstheorie keinen Rational-Choice-Ansatz verwendet). Beispielsweise bewertet er den Einwand einer ,große[n] Erklärungslücke“ seiner situationistischen Perspektive als durchaus legitim und führt weiter aus: „Neben dem, was die situationistische Analyse offenlegt, müssen wir natürlich auch die Hintergrundbedingungen, die sozialen Bedeutungen und das Handlungsrepertoire berücksichtigen, die Menschen dazu bringen, sich feindselig zu begegnen." (ebd., S. $63 \mathrm{f}$.

Im Folgenden wird der situationistische Ansatz von Collins aus seiner erklärungstheoretischen Kritik an den „Mainstreamern“ heraus vorgestellt und gezeigt, dass sich sein Ansatz einer struktur-individualistischen Erklärungsheuristik zuordnen lässt. Diese Zuordnung erfolgt entlang der drei „Logiken“ (der Logik der Situation, der Selektion und der Aggregation) des Grundmodells soziologischer Erklärung und

\footnotetext{
9 Das Modell der soziologischen Erklärung wird von Hobel und Knöbl (2019, S. 134) auf gut einer Seite ad acta gelegt mit der Begründung, es sei insbesondere von Hartmut Esser ,das Verhältnis zwischen Mikro- und Makroebene nicht [...] exakt bestimmt“" worden. Es bleibt aus unserer Sicht unverständlich, weshalb die explanatorische Rückführung von sozialen Phänomenen auf die (mit welchem Mechanismus auch immer) aggregierten Handlungswirkungen, -entscheidungen und Definitionsleistungen der Akteure im Widerspruch zu ,unterschiedlich strukturierten Wirklichkeiten“ stünden. Gerade in den Bänden zu den speziellen Grundlagen der Soziologie werden von Esser (grundlegend 2000b) vielfältige und unterschiedlich strukturierte Wirklichkeiten vermittelt über das Grundmodell der soziologischen Erklärung präsentiert - inklusive der Darlegung möglicher „Eigenständigkeiten“ dieser Phänomene.
} 
verdeutlicht, an welchen Stellen dieses Erklärungsmodells die Warum- und die WieFragen der „Mainstreamer“ und „Innovateure“ ihren Platz finden, sie folglich also keine sich ausschließenden Zugriffe auf Gewaltphänomene darstellen. Zum Schluss wird angedeutet, welche Konsequenzen dies für den Gewaltbegriff hat.

\section{Situationistische Gewaltforschung und das Grundmodell soziologischer Erklärung}

Randall Collins' Vorschlag, die Gewaltforschung müsse sich den „Dynamiken der Gewalt" widmen, hat die aktuelle Gewaltforschung stark beeinflusst (siehe etwa die Beiträge in Mittelweg 36, Heft 1-2, 2019; Equit et al. 2016; Nassauer 2015a, 2015b, 2015c, 2016). Collins' (2011, S. 10 ff.) Behauptung ist, dass Gewaltsituationen nicht hinreichend durch gewaltvorgängige Faktoren - Motive der Täter, strukturelle Faktoren wie Armut, Herkunft etc. - erklärt werden können. Diese Kritik an der explanatorischen Kraft der ätiologischen Forschung begründet Collins' Konzentration auf die Situationen der Gewalt, seine analytische Bevorzugung jener Faktoren, die zeitlich nahe an den Gewalthandlungen liegen und eine unmittelbare Bedeutung für die Interaktionsdynamik haben - was vor allem von den ursachenkritischen Forscher:innen Zuspruch erhielt. Collins' These ist, dass jede Erklärung der Gewalt daran anzusetzen habe, die situative Überwindung der Konfrontationsangst zu untersuchen, weil deren Überwindung notwendig zur Gewaltausübung ist: ,Gewalt läuft gegen eine Barriere aus konfrontativer Anspannung und Angst. Damit Gewalt geschieht, müssen Situationsbedingungen vorhanden sein, die es zumindest einer Seite ermöglichen, diese Barriere zu überwinden." (Collins 2016, S. 18) Mit der Barriere der Konfrontationsanspannung und -angst ist gemeint, dass Akteure grundlegend ein anthropologisch verankertes Bedürfnis nach Verbundenheit und damit an der Aufrechterhaltung der Interaktionsordnung haben (vgl. Collins 2004). Gewalthaltige Situationen gefährden in dieser Perspektive die „,normale“ Interaktionsordnung. ${ }^{10}$ Deshalb scheuen Akteure eher die Konfrontation, entwickeln zudem bei einer Intensivierung der Anspannung die Emotion Angst und erweisen sich selbst dann als überraschend gewaltinkompetent, wenn der strukturelle Kontext eigentlich auf Gewaltanwendung ausgerichtet ist und die Akteure sich zudem mehr oder weniger freiwillig in diesen Kontext begeben haben, um Gewalt auszuüben (etwa im Krieg). Zur Gewaltanwendung sei also die Überwindung der Konfrontationsangst notwendig, was - unter anderem - dadurch erreicht werden kann, dass einer der Akteure emotionale Dominanz gegenüber den anderen Akteuren erzeugt, d.h. , die emotionale Situation zu seinen Gunsten wendet“ (Collins 2011, S. 36). Dieser ,pathway“ wird

\footnotetext{
10 „Gewalttätige Interaktionen sind schwierig, weil sie normalen Interaktionsritualen im Kern zuwiderlaufen. Die Neigung, sich mit dem Rhythmus und den Emotionen des jeweils anderen zu verbinden, hat zur Folge, dass Menschen ein alles beherrschendes Gefühl der Anspannung empfinden, wenn die Interaktion widersprüchlichen Zwecken dient, es sich also um eine antagonistische Interaktion handelt. Dieses Gefühl nenne ich Konfrontationsanspannung; bei wachsender Intensität geht sie in Angst über. Und deshalb fällt uns Gewaltausübung so schwer. Wem sie leichtfällt, der hat einen Weg gefunden, Konfrontationsanspannung und -angst zu umgehen, indem er die emotionale Situation zu seinen Gunsten wendet." (Collins 2011, S. $35 \mathrm{f}$.
} 
von Collins als ,emotional domination, attacking the emotionally weak" (Collins 2017, S. 2) bezeichnet und meint, dass die bereits bestehende emotionale Energie der Interaktion - Anspannung und Angst - in eine emotionale Dominanz überführt werden kann. Collins empfiehlt damit einen Erklärungsansatz, welcher an der aus seiner Sicht notwendigen Bedingung für Gewalt ansetzt: die Erzeugung situativer, d.h. emotionaler Dominanz durch bzw. zwecks Überwindung der Konfrontationsangst. Er geht davon aus, dass diese Prozesse durch „mikro-situative Mechanismen“ bestimmt werden. ${ }^{11}$

Wir bereits weiter oben angedeutet, schließt sich Collins mit seinem situationistischen Ansatz implizit einer struktur-individualistischen Perspektive an, dessen explanatorisches Grundmodell bekanntlich mit dem Modell der soziologischen Erklärung in einer allgemeinen Version bereitgestellt wird. Bedeutsam ist an dieser Stelle, dass dieses Modell häufig im Rahmen von (erweiterten) Rational-Choice-Ansätzen eingeführt, d.h. den Akteuren grundsätzlich ein nutzenmaximierender Handlungsalgorithmus unterstellt wird. In unserem Beitrag soll es allerdings weder primär darum gehen, mit welchen Algorithmen die Akteure wahrnehmen oder zu einer Handlungsentscheidung kommen, noch um die Frage, an welcher Stelle eine „Gesetzmäßigkeit““ vorliegen könnte ${ }^{12}$, sondern ausschließlich um die analytische Differenzierung der Erklärung in drei unabhängige Erklärungsschritte, um die Komplementarität der unterschiedlichen Positionen innerhalb der Gewaltforschung durch einen gemeinsamen Bezugsrahmen zu verdeutlichen. Kurz: Das Erklärungsmodell darf hier nicht mit der in diesem Modell häufig verwendeten Handlungstheorie verwechselt werden.

Das Fundament des Grundmodells soziologischer Erklärung bildet die Annahme, dass man eine Situation zum Zeitpunkt $t_{2}$ nicht direkt aus einer Situation des Zeitpunkts $t_{1}$ ableiten kann. Niemand bezweifelt, dass diese Kernannahme auch für zu erklärende Gewaltsituationen gilt. Zur Erklärung - auch von Gewaltphänomenen sind deshalb drei logisch getrennte Erklärungsschritte zu gehen, welche die Veränderungen der Situation zum Zeitpunkt $t_{2}$ gegenüber der Situation zum Zeitpunkt $t_{1}$ erklärend darlegen: Im ersten Schritt wird die Logik der Situation analysiert, also die Frage, wie die relevanten Akteure die Situation zum Zeitpunkt $\mathrm{t}_{1}$ wahrnehmen und ausdeuten. Im zweiten Schritt wird rekonstruiert, wie die Akteure unter der

\footnotetext{
11 Collins ist an dieser Stelle ungenau und scheint tautologisch zu argumentieren, dass sich Konfrontationsangst durch emotionale Dominanz überwinden lässt. Zugleich heißt es, emotionale Dominanz werde durch die Überwindung der Konfrontationsangst erzeugt - was insgesamt nicht sinnvoll ist. Wir lesen Collins deshalb so, dass die Konfrontationsangst als ein möglicher ,pathway“ Dominanzverhalten auslöst (siehe Miller 2011, S. 120 f.), welches in einem Zuge dazu führen kann, dass der Dominanz zeigende Akteur auch tatsächlich emotionale Dominanz in der Situation erringt. „In einem Zuge“, weil hier Mikroexpressionen wichtig sein dürften: Man richtet sich z.B. im Streit auf und macht sich größer, „,bläht“ die eigene Brust auf, der Kontrahent reagiert darauf, und sei es mit einer Veränderung des Blicks, der womöglich als Schwächemoment gedeutet wird, was wiederum für eine tatsächliche emotionale Dominanz sorgen kann, die dann laut Collins für den Angriff sorgt.

12 Vertreter:innen des Rational-Choice-Ansatzes bevorzugen die Unterstellung eines nutzenmaximierenden Algorithmus zur Selektion von Handlungen und sehen darin jene anthropologisch fundierte Gesetzmäßigkeit, welche die soziologische Erklärung im Sinne des Hempel-Oppenheim-Schemas trage (siehe Esser 1999, S. 204 ff.). Das Modell der soziologischen Erklärung ist mit dieser theoretischen Annahme vereinbar, diese theoretische Annahme ist aber keine zwingende Voraussetzung für die Verwendung des Modells der soziologischen Erklärung.
} 
gegebenen Rahmung der Situation eine Handlung unter verschiedenen Handlungsalternativen auswählen: Es muss die Logik der Selektion rekonstruiert werden, d.h. die im konkreten Fall vorliegende Handlungsentscheidung der Akteure. Im letzten und dritten Schritt wird die Logik der Aggregation ermittelt und das handelnde Zusammenwirken der Akteure rekonstruiert. Das Ergebnis der Aggregation ist die zu erklärende und mit den drei Schritten dann auch erklärte Situation zum Zeitpunkt $\mathrm{t}_{2}$.

\subsection{Logik der Situation}

Wenn Collins davon ausgeht, dass die Hinwendung zur Gewalt ,situativ entschieden“ wird, dann bedeutet dies übersetzt in das Modell der soziologischen Erklärung nichts anderes, als dass die Akteure mittels der von ihnen hervorgebrachten Definition der Situation eine Gewalthandlung auswählen. Collins betont besonders die „emotionale Energie“ bei der Wahl einer Gewalthandlung und geht davon aus, dass es sich nicht um eine kognitiv-rationale Handlungsentscheidung handelt, sondern um die Überwindung unserer, ,physiologischen Programmierung [...], die nach Einbindung in ein mikrointeraktives Ritual strebt“" (Collins 2011, S. 125; vgl. Kron 2020a). Insbesondere Hartmut Esser (2000a) hat sich darum verdient gemacht aufzuzeigen, wie strukturelle und kulturelle Bedingtheiten derartig auf die Orientierung der Akteure einwirken können, dass quasi-automatische Handlungsreaktionen folgen - und nichts anderes sind nach Collins die Hinwendungen zur Gewalt von Akteuren in Situationen hochgradiger Konfrontationsanspannung. Die Wirkung struktureller und kultureller Einflüsse auf die Definition der Situation kann grundsätzlich von einer rein rationalen Abwägung bis hin zu unmittelbar wirkenden Faktoren der Situationsbedingungen reichen. Jene Unmittelbarkeit der Situationsbedingungen meint, dass Akteure automatisch das abrufen, was sie mental in Form von möglichen Situationsdeutungen (Frames) und Handlungsoptionen (Skripte) gespeichert haben. Dies geschieht unter Umständen gänzlich ungestört und ohne Zwischenschaltungen rationaler Überlegungen, wenn die Bedingungen der Situation perfekt mit den verfügbaren mentalen Modellen des Akteurs zusammenpassen (,matchen“). ${ }^{13}$

In Bezug auf die Modellierung von Gewaltsituationen kommt bei Collins der perfekte Match dadurch zustande, dass bestimmte situative Gegebenheiten zum Zeitpunkt $t_{1}$ vorliegen, welche die anthropologisch jedem Menschen gegebene ,Konfrontationsangst" überwinden helfen, insofern sich die Akteure durch die Gegebenheiten in spezifische situative emotionale Zustände versetzen lassen. Eine solche eindeutige Automatik des darauffolgenden Handelns beschreibt Collins (2011, S. 130ff., 2015) als „Vorwärtspanik“, welche dann in den neuen Aggregatzustand zum Zeitpunkt

\footnotetext{
13 Mit diesem Argumentationsstrang modelliert Esser (2003a, 2006) den idealtypischen wertrationalen „homo sociologicus“, der die sozialisatorisch einverseelten normativen Vorgaben unreflektiert in Handeln umsetzt. Ein ähnlicher Strang modelliert das automatische, durch Emotionen gesteuerte Reagieren, den „emotional man“ (vgl. Schimank 2000).
} 
$\mathrm{t}_{2}$ führt. ${ }^{14}$ Nassauer $(2016,2015 \mathrm{c}$; vgl. Collins 2011, S. 185 ff.) erklärt diesen Zusammenhang exemplarisch am Ausbruch kollektiver Gewalt zwischen Polizist:innen und Demonstrant:innen bei an sich friedlichen Demonstrationen, indem sie aufzeigt, wie durch bestimmte kritische Situationsparameter zum Zeitpunkt $t_{1}$ bei den Akteuren emotionale Dynamiken in Gang gesetzt werden, die dann zur Überwindung der Konfrontationsangst sowie zur Erzeugung emotionaler Dominanz bei einer Gruppe und damit zur Selektion von Gewalthandlungen führen. Mit den Situationsbedingungen sind im konkreten Untersuchungsfall das Eindringen in den Raum der jeweils anderen Gruppe (Polizei oder Demonstrierende), organisatorische Schwierigkeiten bei der Polizei und Sachbeschädigungen durch Demonstrant:innen sowie Kommunikationsschwierigkeiten zwischen Demonstrierenden und Polizei gemeint. Diese Situation ist emotional durch einen Anstieg von Anspannung und Angst auf beiden Seiten geprägt. Fühlt sich eine der beiden Gruppen dann im Vorteil emotionaler Dominanz (z.B. weil die andere Gruppe in Unterzahl erscheint), kann sich diese Anspannung und Angst in einem von Vorwärtspanik getriebenen Angriff auf den vermeintlich Schwächeren entladen.

Im Modell der soziologischen Erklärung erfolgt die Klärung der Logik der Situation vor der Klärung der Selektionslogik. Dieser Reihung entspricht Nassauer mit dem Vorschlag, aus „Collins' Ansatz herauszuzoomen und sich nicht rein auf die Emotionen in den Minuten und Sekunden vor der Gewaltentstehung, sondern auch auf die Interaktionen und Interpretationen von Akteuren in den Stunden und Minuten vor der Gewaltentstehung zu konzentrieren“ (Nassauer 2015c, S. 501). Hier zeigt sich die relevante Differenz des situationistischen Ansatzes im Vergleich zu den „Mainstreamern“ in dem für erklärungsrelevant befundenen Zeitausschnitt: Während die Vertreter einer situationistischen Gewaltanalyse einen sehr kurzen Zeitausschnitt vor dem Gewalthandeln als Fokus bevorzugen, gehen die „Mainstreamer“ in der Regel weiter in der Zeit zurück und betrachten dabei allgemeinere - eben strukturelle und kulturelle - ,transsituative“ Parameter. ${ }^{15}$ Geht man noch weiter in der Zeit zurück, könnte man gar evolutionär bedingte neurobiologische Parameter als Teil der Situationslogik in die Erklärung einspeisen (Meyer 2004; Thornhill und Palmer 2000), sofern der theoretische Rahmen der Untersuchung deren Wirkungsweise postuliert. Welcher Zeitausschnitt für soziologische Erklärungen angemessen ist, ist bislang nicht generell geklärt worden, und man darf behaupten, dass dies bestenfalls am jeweils vorliegenden empirischen Fall fruchtbar diskutiert werden kann.

\footnotetext{
14 Vorwärtspanik ist ein Modus zur Entladung der Konfrontationsangst. Einmal aktiviert, ist sie in der Regel nicht mehr aufzuhalten: „Eine Vorwärtspanik ist Gewalt, die nicht zu stoppen ist. Sie ist ein Overkill, ein Kraftaufwand, der weit über das Maß hinausgeht, das für einen Sieg notwendig wäre. Wer aus der Anspannung in eine Vorwärtspanik abrutscht, ist in einen Tunnel eingefahren und kann nicht anhalten." (Collins 2011, S. 145) Sie entsteht ,in einer Atmosphäre totaler Überlegenheit“ (ebd., S. 157).

15 Das weitere Zurückgehen in der Zeit bedeutet jedoch nicht zwingend, dass allgemeinere Situationsparameter berücksichtigt werden (müssen), wie auch die Berücksichtigung eines nur kurzen Zeitausschnittes nicht zwangsläufig auf eine gänzliche Ausblendung allgemeinerer Situationsparamater hinausläuft bzw. hinauslaufen muss.
} 
Noch wahrscheinlicher ist, dass sich unterschiedliche Zeitrahmen berücksichtigende Erklärungen empirischer Fälle komplementär ergänzen können. ${ }^{16}$

Schon dieser knappe Abgleich des allgemeinen Modells der soziologischen Erklärung mit der ,situationalen Gewaltanalyse“ genügt, um einen Großteil der Missverständnisse des Gewaltforschungsdiskurses zu entschärfen. So ist auf der Grundlage dieser Erklärungsschablone z.B. grundsätzlich klar, dass Gewalthandlungen immer auch strukturell und kulturell mitbedingt sind, denn in diesem Modell gibt es überhaupt kein Handeln ohne jegliche strukturelle oder kulturelle Einflüsse. Damit ist die Forderung etwa von Endreß (2014, S. 100) nach einer „,sozial- und gesellschaftsanalytischen Einbindung“ quasi eingepreist. Die erklärungstheoretisch relevante Frage ist, an welcher Stelle und in welcher Form die strukturelle Bedingtheit berücksichtigt wird - keine Frage ist indes, $o b$ diese explanatorische Berücksichtigung finden muss. Im Grundmodell der soziologischen Erklärung ist der Platz zur Berücksichtigung struktureller und kultureller Einflüsse mindestens in der Analyse der Logik der Situation. Wie Nassauer (2015c, S. 510f.) folglich zu Recht anmerkt, bedeutet die Konzentration auf jene die Konfrontationsangst beeinflussenden kurzfristigen Situationsbedingungen nicht, dass strukturelle und kulturelle Faktoren explanatorisch überhaupt keine Rolle spielen würden. Wenn es darum geht, Gewalthandeln zu erklären, dann können die strukturellen und kulturellen Randbedingungen relevante Parameter der Erklärung sein, z.B. um die Definition der spezifischen Situation zum Zeitpunkt $t_{1}$ darzulegen, welche dann zur Vorwärtspanik geführt hat. Und auch die Beschreibung einer Situation als ,reziproke Sinn- und Deutungsprozesse“ (Endreß 2004, S. 199) kann als Teil der Situationsanalyse zur Aufklärung der sozialen, sachlichen, zeitlichen und räumlichen Bedingungen von Gewalthandlungen beitragen. Zugleich ist Darlegung der Situationslogik inklusive möglicher struktureller und kultureller Faktoren natürlich noch keine abschließende Erklärung des untersuchten Gewaltphänomens. Anders formuliert: Mit einer solchen Situationsanalyse ist nicht festgelegt, dass ,die strukturelle Öffnung des objektiven Möglichkeitsraums des Handelns das den Gewaltexzessen zugrundeliegende Phänomen darstellt, um die es der Analyse von Gewaltverhältnissen gehen muß“ (ebd., S. 180; Hervorh. T. K. u. L. V.). Für eine vollständige Erklärung muss es neben der Rekonstruktion der Situationslogik ebenso um die Rekonstruktion der Logik der Selektion und der Aggregation gehen.

\subsection{Logik der Selektion}

Der nächste Schritt im Erklärungsmodell ist die Analyse der Logik der Selektion, die - immer vor dem Hintergrund der Situationslogik - den Fokus auf die Wahl einer Handlung aus einer Alternativmenge von Handlungsmöglichkeiten legt. Auch ein automatisches Reagieren, etwa das unreflektierte Umsetzen internalisierter normati-

\footnotetext{
16 Dies begründet u.a. die Nähe jener, die Erklärungen mittels sozialer Mechanismen finden wollen, zur historischen Soziologie, zusammengeführt etwa in dem Konzept der Pfadabhängigkeiten, das den Zusammenhang von Prozessualität und Historizität als wesentlichen Bestandteil mechanismischer Erklärungen herausstellt (siehe exemplarisch Mahoney 2000).
} 
ver Vorgaben ${ }^{17}$ oder emotionsgeleitete Handlungsentscheidungen, ist als spezifischer Modus der Handlungsselektion zu verstehen. Automatisches Reagieren bedeutet, bestimmte Wahrnehmungs-, Deutungs- und Selektionsvorgänge bereits derart gelernt und verinnerlicht zu haben, dass die Handlung unhinterfragt in einer Situation selegiert und eben nicht in jeder Situation einer Reflexion unterzogen wird: Nicht nur die Selektion der dann tatsächlich gewählten Handlungsalternative, auch die Wahl des Selektionsalgorithmus ${ }^{18}$ selbst kann automatisiert erfolgen. Die Selektion einer Handlung kann also „,abgekürzt“ werden, sofern Akteure im Rahmen des perfekten Matchings zwischen Situationsdeutung und Handlungsoption (etwa bei Routinen oder Ritualen) automatisch bzw. prompt reagieren und agieren. Die Erklärung einer Handlung ist nur dann vollständig, wenn auch die Handlungsselektion rekonstruiert wurde - eine situationistische Erklärung von Gewalt im Sinne von Collins benötigt somit die Rekonstruktion der Selektionslogik für eine gewaltsame Handlung durch Darlegung der Mechanismen der Überwindung der Konfrontationsangst sowie der Erzeugung emotionaler Dominanz.

Auch die Rekonstruktion der Selektionslogik für Collins' Ansatz kommt nicht ohne Kontextualisierung aus. Ob eine spezifische situative Konstellation zum Zeitpunkt $\mathrm{t}_{1}$ zur Überwindung der Konfrontationsanspannung und zu emotionaler Dominanz führt, ist nämlich durchaus abhängig vom jeweiligen strukturellen und kulturellen Kontext dieser Konstellation: Vergleichbare Situationen mögen in dem einen Fall etwa in Vorwärtspanik resultieren, in anderen Fällen aber eventuell eher zur Flucht, zur Schockstarre oder zu anderen „menschlichen“ Verhaltensweisen in Konflikten wie z.B. Dominanzverhalten oder Unterwürfigkeit (Miller 2011, S. 120f.) führen. Die Ausprägung der westlichen Kultur beinhaltet in Deutschland beispielsweise die Vorstellung, dass die innerliche Freiheit des Einzelnen von außen durch den Staat geschützt werden müsse (Münch 1986). Gewalt soll folglich möglichst aus dem Privaten herausgehalten und bei Polizei und Militär als Mittel zum Schutz der Verteidigung der individuellen Freiheiten verortet werden. Selbstschutz angesichts einer Konfliktsituation, die in Gewalt enden könnte, bedeutet z.B. dann, die Polizei zu involvieren, was die Wahrscheinlichkeit situativer Vorwärtspanik verringern dürfte. ${ }^{19}$ Die US-amerikanische Variante der modernen Kultur sieht dagegen die Freiheit des Einzelnen in den sozialen Bezügen aufgehen, weshalb es so wenige Einflüsse wie möglich von außen geben dürfe. Selbstschutz beinhaltet dann folgerichtig das Recht, sich selbst schützen zu können, die eigene Freiheit im sozialen Bezug herzustellen, indem man zum Selbstschutz etwa selbst zur Schusswaffe greift. Während in Deutschland der Besitz und das Mitführen von Schusswaffen folglich nur als Ausnahme erlaubt ist, ist es in den USA ausdrücklich gestattet und geradezu Ausdruck dieses spezifischen Individualismus, sich ggf. auf diese effektive Art und

${ }^{17} \mathrm{Zu}$ diesen normativen Vorgaben gehören auch „Verfahrensordnungen“ (Lindemann 2017).

18 Als Algorithmen von Handlungsentscheidungen stehen etwa „Bauchentscheidungen“ oder andere Heuristiken (Gigerenzer und Todd 1999; Gigerenzer 2007) genauso zur Verfügung wie die Identitätsbehauptung (Schimank 2000, S. 121 ff.) oder Planung, Im-Spiel-Bleiben und Inkrementalismus (Schimank 2005).

19 Das sogenannte „Aachener Modell“ der Gewaltprävention - ein von der Polizei Aachen zusammen mit der Unfallkasse NRW eingeführter Präventionsleitfaden für den Öffentlichen Dienst und ähnliche Institutionen - fasst dies mit dem Merksatz zusammen: „Sicherheit muss von Profis hergestellt werden!“ Verhindert werden soll dadurch, dass Beschäftigte zu viel Selbstschutz praktizieren. 
Weise verteidigen zu können. Akteure, die diese unterschiedlichen Deutungsmuster internalisiert haben, werden vermutlich entsprechend anders auf Angriffe reagieren, nämlich eher mit Flucht auf der einen (Deutschland) und mit Angriff auf der anderen Seite (USA).

Derart bestimmen kulturelle und strukturelle Muster also ebenfalls mit, welche Ereignisse situativ geeignet sind, um z.B. eine Vorwärtspanik auszulösen. Wenn in den USA im Jahr 2015 ein Höchststand erreicht wurde an jungen schwarzen Männern, die durch Polizisten erschossen wurden (Swaine et al. 2015), dann gilt der in den USA verwurzelte Rassismus gegenüber Schwarzen als ein wesentlicher kultureller Erklärungsfaktor - der gleichwohl für sich als Erklärungsfaktor nicht hinreichend ist. Und auch zur Erklärung der Gewalt von israelischen Soldaten gegenüber palästinensischen Terroristen - zur Erklärung, warum die meisten Terroristen in Israel getötet (,neutralisiert“) und nicht verhaftet werden - wird man kulturelle, insbesondere historisch-religiöse Randbedingungen genauso wie politische Bedingungen in der wechselseitigen Wahrnehmung für eine vollständige sozialwissenschaftliche Erklärung wohl berücksichtigen müssen. Ähnliches dürfte sich für verschiedene Subkulturen feststellen lassen: Rockergruppen, Kampfsportler oder Kirchenchorsänger dürften durchaus unterschiedliche Schwellen und Trigger zur Überwindung ihrer Konfrontationsangst aufweisen (vgl. Staack 2015).

Auch ein enger Gewaltbegriff entlastet nicht von dem explanatorischen Einbezug struktureller (oder kultureller) Bedingungen für die Erklärung von Gewaltphänomenen. ${ }^{20}$ Wichtig für den Gewaltforschungsdiskurs ist die Verortung der strukturellen und kulturellen Bedingungen als Teil der Gesamterklärung von Gewaltphänomenen, ohne den Gewaltbegriff an sich mit Kultur oder Struktur aufzuladen und damit explanatorische Kurzschlüsse zu suggerieren. Die Begriffe von „kultureller“, ,struktureller“" oder ,symbolischer“ Gewalt selbst haben keine eindeutigen erklärungstheoretischen Implikationen, sondern die Adjektivbildung markiert eine Präferenz für eine bestimmte Dimension bzw. für eine bestimmte Gestalt des Gewaltphänomens, hinter der dann eine spezifische Logik der Gewaltausübung und Gewaltverursachung stecken mag, dessen Analyse wiederum Teil der Erklärung ist. Dementsprechend ist Collins zuzustimmen, dass strukturelle oder kulturelle Faktoren Gewalthandeln allein nicht hinreichend erklären können.

Bei Collins wird allerdings ebenso wie bei den „Mainstreamern“ und anderen „Innovateuren" die Unterscheidung von notwendigen und hinreichenden Bedingungen nicht expliziert. Diese Unterscheidung ist für soziologische Erklärungen aufgrund der Komplexität sozialer Phänomene und der unterschiedlichen kausalen Relevanz der involvierten Bedingungen zentral (Ragin 2000) und kann an dieser Stelle zur weiteren Aufschlüsselung der Collins'schen Argumentation herangezogen werden. ${ }^{21}$

\footnotetext{
20 Sutterlüty (2015, S. 241) sieht genau an dieser Stelle eine Grenze situationistischer Analysen, weil sich die normativen Erwartungen, mit denen die Akteure in die Situationen geraten, nicht aus den Situationen selbst ableiten lassen, wie er am Beispiel der „Riots“ in den USA demonstriert, bei denen die ethnischen Minderheiten die „Idee staatsbürgerlicher Gleichheit voll internalisiert“ haben.

21 Nassauer (2015c, 2012) stellt diese Unterscheidung von notwendigen und hinreichenden Bedingungen zwar für ihre empirische Untersuchung voll in Rechnung, indem sie als Methode die Qualitative Comparative Analysis (QCA) verwendet (siehe Ragin 1986). Eine erklärungstheoretische Berücksichtigung erfährt diese Unterscheidung bei ihr jedoch nicht.
} 
Bedingungen sind dann notwendig für ein soziales Phänomen, wenn die Bedingungen jedes Mal zusammen mit dem betreffenden Phänomen auftreten. Bezogen auf Collins' These zur Gewalt könnte man formulieren: Die Überwindung der Konfrontationsangst ist eine notwendige Bedingung der Gewaltausübung, denn jedes Mal, wenn Gewalt ausgeübt wurde, ist die Konfrontationsangst überwunden worden, und wenn sie nicht überwunden worden ist, kommt es auch nicht zur Gewaltanwendung. Folgerichtig zeigt Collins, wie die Konfrontationsangst in verschiedenen empirischen Situationen zuvor überwunden wurde, wenn es de facto zur Gewaltanwendung gekommen ist. Zudem führt er viele Beispiele an, wo ein gewaltsames Handeln von Akteuren gefordert oder erwartet wurde, es aber nicht dazu kam, weil die Konfrontationsanspannung und -angst nicht überwunden werden konnte. Im militärischen Kampf etwa führt die verbreitete Konfrontationsangst oft zu einer Nichterfüllung der von den Soldaten geforderten Leistung, wie sich an der erstaunlich niedrigen Schieß- und Trefferquote zeigt (Collins 2011, S. 70 ff.).

Mit dieser These der Überwindung der Konfrontationsanspannung und -angst als einer notwendigen Bedingung von Gewalthandlungen ist nicht gesagt, dass diese Überwindung auch eine hinreichende Bedingung für die Gewaltanwendung ist. Eine Bedingung gilt dann als hinreichend, wenn ihr Auftreten das betreffende soziale Phänomen mit Notwendigkeit nach sich zieht. Mitnichten führt jedoch die Überwindung von Konfrontationsangst immer auch zur Gewaltanwendung: So besteht die Kompetenz von Türstehern gerade darin, nicht auf Provokationen der Gäste mit körperlicher Gewalt zu reagieren, obwohl man unterstellen darf, dass die grundsätzlich vorhandene Konfrontationsangst bei diesen professionalisierten Akteuren rasch überwunden und die emotionale Dominanz im Regelfall gegeben ist. Es ist der juristische, berufsethische und geschäftliche Auftrag, als Türsteher (oder „Security“) „,besonnen“ auf Provokationen zu reagieren und von körperlicher Gewalt solange wie möglich abzusehen, auch wenn Gewalt als Thema dort sicherlich überproportional häufig kommuniziert wird (Preiser 2016).

Vor diesem Hintergrund lässt sich die Kritik von Collins an den „Mainstreamern“ sowie sein situationistischer Gegenvorschlag evaluieren: Strukturelle Bedingungen von Gewalt sind handlungsprägend und nicht handlungsfähig (Schimank 1985), Strukturen üben keine Gewalt aus. Ob Strukturen - wie etwa der Begriff der ,,strukturellen Gewalt" suggeriert - hinreichend zur Erklärung von Gewalt sind, müsste die jeweilige Erklärung erst nachweisen; sie können es empirisch sein, müssen es aber nicht. Hier setzt Collins' Kritik an: „Being a racist is not sufficient to make someone violent; nor is being poor, subaltern, shamed, hopeless, angry, or filled with religious fervor." (Collins 2012, S. 135) Aus diesem Grund rückt Collins die notwendigen Bedingungen von Gewalt in den Vordergrund. Der danach folgende Satz verdeutlicht allerdings Collins' Vernachlässigung von Bedingungen, welche möglicherweise (in Kombination mit notwendigen Bedingungen) hinreichend sind: „It does not matter [sic!] how angry or alienated someone is; they still have to get past the barrier of confrontational tension." (ebd.) Der epistemologische Fehlschluss bei Collins ist eindeutig: Weil eine Bedingung - die Überwindung der Konfrontationsanspannung notwendig ist, spielen andere Faktoren keine Rolle.

Es ist für den Gewaltforschungsdiskurs lohnenswert, sowohl die Unterscheidung von notwendigen und hinreichenden Bedingungen für das Gewalthandeln explana- 
torisch zu berücksichtigen als auch die analytische, für die Erklärung wichtige Differenz zwischen der Logik der Situation und der Logik der Selektion zu erkennen. ${ }^{22}$ Denn dadurch lässt sich verdeutlichen, dass Strukturen einen maßgeblichen, wenngleich verschiedenartigen Einfluss auf das Gewalthandeln haben (nämlich in Form von unterschiedlichen Beiträgen zu notwendigen und hinreichenden Bedingungskonfigurationen). Die Strukturen der Ausgangssituation sind explanatorisch zu berücksichtigen als handlungsprägende Strukturen - als handlungsermöglichende bzw. als bestimmte Handlungen nahelegende Strukturen -, aber sie allein vermögen die konkrete Hinwendung zum Gewalthandeln (noch) nicht zu erklären. Anders formuliert: Selbstverständlich gibt es empirisch Situationen, deren Strukturen eine hochgradige Wahrscheinlichkeit innewohnt, dass Gewalt als Handlungsoption selegiert wird. Beispielsweise impliziert die Struktur der Intimbeziehungen in Deutschland aktuell eine höhere Wahrscheinlichkeit für Frauen, physische Gewaltopfererfahrungen mit (Ex-)Partnern zu machen als mit fremden Personen im Rahmen der Struktur des öffentlichen Lebens und öffentlicher Orte. ${ }^{23}$ Allerdings führt eine solche strukturelle Prägung eben nicht zwangsläufig zu Gewalt. Selbst eine als „Krieg“ definierte soziale Struktur (Rotte 2019) muss nicht zwingend zu Gewalt führen, sondern kann zu kooperativ-friedlichen Interaktionen führen (Axelrod 2000, S. 67 ff.). Ein Begriff wie ,strukturelle Gewalt“ verkürzt die Erklärung durch die Suggestion einer Unbedingtheit, so als würden bestimmte Strukturen ,immer“ zu Gewalthandeln führen. Semantische Ausweichbewegungen wie die (richtige) Feststellung, dass die ,symbolische Ordnung des Staats nicht selbst schon Gewalt“ sei, ,,aber die gewaltsamen Effekte ihr deutlich eingeschrieben [...] sind“ (Imbusch 2017, S. 49), zeigen nur allzu deutlich die Mängel einer Erklärungsheuristik, die nicht sauber zwischen Situations-, Selektions- und Aggregationslogik trennt. Man muss immer grundsätzlich die Logik der Selektion darlegen.

Wir können festhalten: Erstens ist nach Collins die Überwindung von Konfrontationsangst eine notwendige Bedingung zur Gewaltausübung, deshalb muss eine Erklärung von Gewaltereignissen diese Bedingung mit einbeziehen. Zweitens ist die Überwindung von Konfrontationsangst keine hinreichende Bedingung für Gewalthandlungen: Gewalt ist zwar ein allen Menschen zugängliches Handlungspotenzial, eine stets vorhandene „Aktionsmacht“ (Popitz 1992, S. 48), aber ob diese Option gezogen wird oder nicht, ist entscheidungsoffen, und folglich muss in der soziologischen Erklärung dieser Entscheidungsprozess der Akteure rekonstruiert werden. Damit gilt drittens: Aus einer bestimmten Struktur kann nicht unmittelbar abgeleitet werden, dass die Akteure Gewalt als Handlungsoption selegieren. Und selbst wenn sich die Akteure für Gewalthandlungen entscheiden, liegt das soziologische Explanandum letztlich auf der sozialen Aggregationsebene, sodass man, viertens,

\footnotetext{
22 Das Grundmodell der soziologischen Erklärung selbst unterscheidet nicht zwischen notwendigen und hinreichenden Bedingungen.

23 In der durch das BMFSFJ herausgegebenen Studie „Lebenssituation, Sicherheit und Gesundheit von Frauen in Deutschland“ (2004, S. 46) gaben 19,5\% der Frauen mit Opfererfahrungen durch physische Gewalt an, dass ihnen die Täter unbekannt waren, während 50,2\% (Ex-)Partner:innen und 30,1 \% jemand aus der Familie nannten (Mehrfachnennungen waren möglich). Ähnlich lagen die Verhältnisse bei sexualisierten Gewaltopfererfahrungen (ebd., S. $78 \mathrm{f}$.).
} 
das handelnde Zusammenwirken der (gewalttätigen und gewalterleidenden) Akteure $\mathrm{zu}$ rekonstruieren hat.

\subsection{Logik der Aggregation}

Aus einer bestimmten sozialen Situation zum Zeitpunkt $t_{1}$ kann analytisch nicht unmittelbar abgeleitet werden, dass zu einem Zeitpunkt $t_{2}$ weitere Strukturen entstehen, die den Folgen von Gewalthandlungen ähnlich sind (Leid, Schmerzen, Elend, etc.). Diese aus dem Modell der soziologischen Erklärung abgeleitete Kritik an den „Mainstreamern“ verweist auch auf die oftmals vernachlässigte Explikation der Logik der Aggregation. Während diejenigen Forscher:innen, die sich dem situativen Ansatz im Anschluss an Collins verschrieben haben, die Aggregationsdynamiken als ,mikrointeraktionalen Verstrickungen“ rekonstruieren und wie Nassauer (2015c) Gewaltereignisse als Ergebnis des handelnden Zusammenwirkens von Polizisten und Demonstranten unter bestimmten situativen Randbedingungen konzipieren, vermisst man bei den Vertreter:innen einer strukturellen oder kulturellen Gewaltkonzeption oftmals eine Explizierung dieser Aggregationsprozesse. Suggeriert wird mit dem Begriff der ,,strukturellen Gewalt" etwa der direkte Durchgriff von sozialen Strukturen zum Zeitpunkt $t_{1}$ - vermittelt über unterschiedliche, auch nicht-gewalttätige Arten des Handelns - auf das Aggregationsergebnis (z.B. kollektive Gewalt) zum Zeitpunkt $t_{2}$. Gemessen an der Unterscheidung von notwendigen und hinreichenden Bedingungen bleibt allerdings unklar, was genau der Begriff „strukturelle Gewalt“ an dieser Stelle der Erklärung ausdrücken möchte: Wird von ,struktureller Gewalt“ gesprochen, da bestimmte Strukturen immer vorliegen müssen, damit eine bestimmte Art von Gewaltaggregat entsteht? So wäre etwa Zygmunt Baumans (1989, 1991) Erklärung des Holocaust zu lesen, welcher die Struktur bzw. die „gärtnerische“ Kultur der Moderne zur notwendigen Bedingung für den Holocaust erklärt hat. Allerdings ist die moderne Gesellschaft - man möchte sagen: glücklicherweise - keine hinreichende Bedingung für den Holocaust. Eine solche notwendige Struktur müsste im Rahmen des Modells soziologischer Erklärung als Teil der Situationsanalyse ermittelt und dann noch gezeigt werden, welche Bedingungen empirisch hinzukommen, damit über das handelnde Zusammenwirken der Akteure tatsächlich das zu erklärende Gewaltaggregat entsteht. Eine starke strukturelle Erklärung von Gewalt würde demnach aufzeigen, dass eine bestimmte Struktur immer zu Gewaltaggregaten führt und in diesem Sinne eine hinreichende Bedingung für ihre Entstehung ist. Unseres Wissens nach sind derartige Strukturen bislang nicht gefunden worden, weil es in komplexen, selbstkritikalen (Kron und Grund 2009), durch Transintentionalität (Greshoff et al. 2003) gekennzeichneten Gesellschaften unwahrscheinlich ist, dass eine bestimmte soziale Struktur immer bestimmte Folgen zeitigt, die den Konsequenzen von Gewalthandlungen ähnlich sind oder nicht.

Es ist nicht zu sehen, bei welchen Strukturen zum Zeitpunkt $t_{1}$ empirisch immer ein bestimmtes Gewalt-Aggregationsergebnis zum Zeitpunkt $t_{2}$ erzeugt wird. Schroer (2004, S. 164 f.) stellt zu dieser Gleichsetzung einer Ausgangsstruktur zum Zeitpunkt $t_{1}$ mit einer Gewalthandlung zum Zeitpunkt $t_{2}$ die Frage, ob „Armut und Abhängigkeitsverhältnisse im Nord-Süd-Konflikt etwa letztlich nicht auf das Töten anderer Menschen hinaus[laufe]“. Es ist offenkundig, dass dieses Gleichsetzen 
der Situation zum Zeitpunkt $\mathrm{t}_{1}$ (inklusive Armut und Abhängigkeit) mit den aggregierten Konsequenzen zum Zeitpunkt $t_{2}$ (Töten anderer Menschen) fehlläuft, denn grundsätzlich endet Armut genauso wenig zwingend im Tod wie andere Abhängigkeitsverhältnisse. Letztlich liefe diese Argumentation darauf hinaus, dass das Leben in Strukturen tödlich ist - eine soziologisch unbefriedigende Erkenntnis, sofern man diese nicht weiterführt (wie z.B. Simmel 1922, S. 96 ff.).

Damit soll selbstverständlich nicht bezweifelt werden, dass es Strukturen sozialer Ungleichheit gibt, die Mehrheit der reichen Industrieländer im globalen Norden, die meisten armen Entwicklungsländer im globalen Süden liegen und dies im Ergebnis für viele Menschen im globalen Süden ein elendiges und ein - gemessen an der Lebenserwartung reicher Länder - vorzeitig beendetes Leben bedeutet. Eine soziologische Erklärung hat jedoch zu rekonstruieren, wie ein solcher Aggregatzustand zum Zeitpunkt $t_{2}$ als Ergebnis des handelnden Zusammenwirkens von kollektiven und korporativen globalen Akteuren zustande kommt, wenn Letztere spezifische Handlungsentscheidungen unter gegebenen (wirtschaftspolitisch-)strukturellen und (westmodern-)kulturellen Randbedingungen zum Zeitpunkt $t_{1}$ treffen (siehe Lessenich 2016). Auf diese Weise kann eine im Rahmen des Modells der soziologischen Erklärung geführte Erklärung z.B. aufzeigen, wie die Implementation des Paragraphen $\S 16$ e in das Zweite Buch des Sozialgesetzbuches - die Einführung eines Beschäftigungszuschusses zur dauerhaften Beschäftigungsförderung von zuvor Langzeitarbeitslosen - letztlich im handelnden Zusammenwirken der verschiedenen Akteure scheiterte und zu leidvergrößernden enttäuschten Erwartungen bei vielen Akteuren führte (Bauer et al. 2012). Das Gesetz als solches bedingt aber weder die Handlungswahlen der relevanten Akteure noch das kollektive Leid unmittelbar zwingend und hinreichend.

Eine (erschöpfende) Erklärung von Gewaltereignissen ist erst dann geleistet, wenn der transformierende Mechanismus ${ }^{24}$ analysiert wurde, unter welchen zusätzlichen (ggf. kulturellen oder strukturellen) Bedingungen die Überwindung von Konfrontationsangst hinreichend zum Übergang in Gewaltaggregate durch das handelnde Zusammenwirken der Akteure ist. Es müssen phänomenspezifisch die Kombinationen der notwendigen und/oder hinreichenden Bedingungen aufgeführt und in ihrer prozessualen Entwicklung ,,seziert“ (Hedström 2005) werden. ${ }^{25}$ Collins (2016, S. 17 ff.) selbst konzentriert sich in seinem Modell auf die Darlegung der Handlungsselektion als Entstehung von Konfrontationsanspannung über emotional-soziale Mechanismen sowie ihrer situativen Überwindung im Rahmen von ,,mikrointeraktionalen Verstri-

\footnotetext{
${ }^{24}$ Hedström und Swedberg (1998a, S. 21 ff.) unterscheiden dementsprechend in Anlehnung an das Modell soziologischer Erklärung drei Typen von Mechanismen: (1) „situational mechanisms“, die sich auf die Einflüsse ,objektiver“ Gegebenheiten von Situationen auf die Akteure, also auf die Makro-MikroVerknüpfung beziehen; (2) ,action-formation mechanisms“, die sowohl die Definition der Situation der Akteure als auch die Umsetzung in das tatsächliche Handeln in den Blick nehmen, und (3) ,transformational mechanisms“, die das handelnde Zusammenwirken der Akteure thematisieren.

25 Die von Hoebel und Knöbl (2019) präferierte prozesssoziologische Betrachtung des Handlungsablaufs kann durch die Verkettung von mehreren Grundmodellen abgebildet werden: Die Aggregation zum Zeitpunkt $t_{2}$ wäre als Ausgangspunkt für eine weitere Situation (und den damit verbundenen Logiken der Situation, Selektion und Aggregation) zu modellieren - man würde damit nicht nur der Zeitlichkeit Rechnung tragen, sondern ebenso die Randbedingungen in die Erklärungsheuristik einspeisen. Zur Abbildung sozialer Prozesse mithilfe des Grundmodells soziologischer Erklärung siehe z.B. Esser (1999, S. 17f.).
} 
ckungen“ - und vernachlässigt in der Regel die weitere Erklärung der sozialen Aggregation.

Gerade aufgrund der zumeist nicht hinreichend eindeutigen sozialen Zusammenhänge zwischen den Ausgangsbedingungen und der aggregierten Gewalt besteht die soziologische Erklärungskunst darin, den Mechanismus ihres Entstehungszusammenhangs zu entschlüsseln und derart das kollektive Gewaltphänomen zu erklären. Ist der Mechanismus dieses spezifischen Zusammenhangs aufgedeckt, erlaubt dessen Erkennen dann ggf., gezielte Interventionen einzuführen. Der situative Ansatz von Collins kann solche Interventionspunkte ausschließlich in der situativen Stabilisierung der Konfrontationsangst finden (siehe Collins 2011, S. 700 ff.). Strukturelle oder kulturelle Präventionsmöglichkeiten bzw. Interventionen, die auf das handelnde Zusammenwirken der bereits gewalttätigen Akteure zielen, um etwa Gewaltspiralen zu unterbinden (Brücher 2011), geraten dadurch aus dem Blick.

\section{Schluss}

Wir möchten in diesem Beitrag den Standpunkt vertreten, dass der Diskurs um den Begriff der Gewalt keinen nennenswerten Fortschritt für die Gewaltforschung mit sich führen wird, solange sich das argumentative Hin und Her um die Begrifflichkeit dreht, ohne die Auseinandersetzung auf der Ebene der Erklärungsmodelle zu suchen. Es ist an der Zeit, wie auch Knöbl (2017, S. 11) resümiert, ,den vielfach geforderten Mikro-Makro-Link tatsächlich herzustellen“. ${ }^{26}$ Dazu ist es allerdings erforderlich, jene Erklärungsmodelle zu benennen, welche den Mikro-Makro-Link - die Verbindung der Prägung des Handelns der Akteure durch die Situation mit ihrer Handlungsentscheidung und dem Ergebnis des handelnden Zusammenwirkens der Akteure - herstellen sollen, denn bekanntlich gibt es dazu mehrere Alternativen (vgl. Alexander et al. 1987; Greve et al. 2009). ${ }^{27}$

Je nachdem, welcher explanatorische Bezugsrahmen gewählt wird, löst sich die Spannung zwischen den „Mainstreamern“ mit der Betonung ,struktureller Gewalt“ und den „Innovateuren“ mit der Konzentration auf einen engen Gewaltbegriff mehr oder weniger auf. Zugleich wird die Provokation durch Randall Collins und seiner geforderten „situationalen Gewaltforschung“ entschärft, die Konzentration der Gewaltforschung auf Strukturen und Motive könnte ,nichts“ zu einer Erklärung des Gewalthandelns beitragen.

\footnotetext{
26 Wobei Knöbl in dem Aufsatz selbst keine Erklärungsmodelle, sondern mögliche Zugänge (Phänomenologie, Ethnomethodologie) entlang verschiedener Analysekategorien (z.B. Gewalträume) diskutiert.

27 In der Konsequenz stimmen wir folglich voll mit Sutterlüty (2015, S. 256) überein: „Eine überzeugende Theorie kollektiver Gewalt muss die analytischen Mittel bereitstellen, die den beträchtlichen Bereich dessen zu fassen erlaubt, was sich nur aus der situativen Dynamik erklären lässt. Zugleich darf sie die Augen nicht davor verschließen, wodurch die entsprechenden Situationen und deren Deutung durch die Akteure ihrerseits bedingt sind. Sie muss - solange sie sich mit dem Gewalthandeln befasst - vor allem erkennen lassen, wie kulturelle Deutungsmuster und sozialstrukturelle Bedingungen situativ handlungsrelevant werden. Die Situation muss, mit einem Wort, als Explanans wie auch als Explanandum Berücksichtigung finden." Sutterlüty (2020; 2015, S. 248) selbst scheint das Modell der soziologischen Erklärung mit seinen drei Schritten am Beispiel der Erklärung von ,urbanen Riots“ oder von Gewalt in der Familie anzuwenden, verzichtet aber auf explizite Hinweise darauf.
} 
Wenn die Erklärung von Gewalt beinhaltet, den Mechanismus (Hedström und Swedberg 1998b; Schmid 2006) zu entschlüsseln, der ausgehend von einer Situation zum Zeitpunkt $\mathrm{t}_{1} \mathrm{zu}$ den Handlungen der relevanten Akteure sowie zur Aggregation eines Gewaltgeschehens zum Zeitpunkt $\mathrm{t}_{2}$ geführt hat, dann wird man nicht darum herumkommen, fallspezifisch den untersuchten sachlichen, zeitlichen und sozialen Ausschnitt anzupassen und ggf. mal enger, mal weiter zu fassen. Die Konzentration ausschließlich auf jene Situationsbedingungen, welche Einfluss auf die aktuelle emotionale Dynamik der beteiligten Akteure nehmen, ist für eine soziologische Erklärung bestimmter empirischer Fälle in der Regel genauso wenig ausreichend wie die reine Beschreibung des Gewalthandelns oder der ausschließliche Hinweis auf strukturelle oder kulturelle Faktoren. Keiner dieser Ansätze genügt gemessen am struktur-individualistischen Grundmodell der soziologischen Erklärung für sich allein einer soziologischen Erklärung. Akzeptiert man, dass eine soziologische Erklärung sozialer Phänomene über Mechanismen erfolgen sollte, bedeutet das zu zeigen, wie ein sozialer Zustand zu einem Zeitpunkt $t_{2}$ sich über dazwischenliegende Schritte (des Handelns und des handelnden Zusammenwirkens von Akteuren) aufgrund bestimmter Bedingungen aus einem bestimmten sozialen Zustand zu einem Zeitpunkt $t_{1}$ herausgebildet (aggregiert) hat (siehe Kron 2006). Es reicht weder aus, die ,ursächlichen“" Faktoren zu beschreiben, ohne den Weg zu erläutern, der von diesen „Ursachen“ zum Explanandum führt, noch genügt eine Beschreibung eines situativen Prozessverlaufes ohne die Angabe der Randbedingungen.

Zum Schluss seien einige Rückschlüsse für den Gewaltbegriff aus der Perspektive des Modells soziologischer Erklärung nur angedeutet: Die Rede von „struktureller Gewalt" ist in diesem explanatorischen Rahmen obsolet, weil jede Erklärung immer fallspezifisch die strukturellen Kontexte berücksichtigen muss, welche die Orientierung der Akteure beeinflussen und derart auf die Handlungsentscheidungen einwirken. Ebenso sind soziale Strukturen explanatorisch in ihrem aggregierenden Einfluss auf das handelnde Zusammenwirken der Akteure zu berücksichtigen. Auch hier wird kein Begriff ,struktureller Gewalt“ benötigt. Aufgrund der genannten Probleme, die mit dem Begriff ,,struktureller Gewalt“ einhergehen und dessen heuristischen Nutzen konterkarieren, liegt es nahe, diesen Begriff gänzlich fallenzulassen.. ${ }^{28}$ Ganz ähnlich dürfte das Argument zur Vermeidung des Begriffs „kultureller Gewalt“ an-

\footnotetext{
${ }^{28}$ So auch Riekenberg (2008, S. 177): Um Gewalt zu erklären, sei „der Begriff der ,strukturellen Gewalt ' jedoch wenig hilfreich. [...] wir müssen uns darüber klar sein, welche Erkenntnisziele wir mit den Begriffen, die wir benutzen, verfolgen wollen. Und die Vorstellung einer ,strukturellen Gewalt ' führt uns auf gänzlich andere Wege als die Absicht, Gewalthandlungen von Menschen in ihren lokalen Kontexten zu verstehen und die ,Sozialität" der Gewalt zu begreifen." In ähnlicher Weise sieht Nungesser in dem von Bourdieu stark gemachten Begriff der ,symbolischen Gewalt“ einen „,erkenntnisbehindernden Pleonasmus“ (Nungesser 2017, S. 21), der die Begriffe Herrschaft, Macht und Gewalt nicht angemessen differenziert, sondern bewusst vermengt. Nungesser vermutet, dies geschehe zum einen aufgrund des ,normativen Grundimpuls[es]“ (ebd., S. 25) in Bourdieus Werks und zum anderen aufgrund einer gewollten „herrschaftsanalytische[n] Entdifferenzierung von Mikro- und Makroebene“ (ebd., S. 26) als eine Form der Theorieentscheidung mit der Konsequenz, dass die „Inflationierung des Gewaltbegriffs und die [...] intentionale und phänomenale Gegenläufigkeit zu dem, was üblicherweise unter Gewalt verstanden wird, [...] zu einer unklaren Verhältnisbestimmung von physischen und symbolischen Gewaltphänomenen [führen] und $[\ldots]$ in der Folge auch die Entwicklung einer historischen Vergleichsperspektive sowie eines normativen Maßstabes [erschweren]“ (ebd., S. 27).
} 
gelegt sein: Weder Strukturen noch Kulturen sind handlungsfähig. Sie können keine Gewalt ausüben. Kulturen können aber ebenso wie Strukturen wesentliche Faktoren einer Erklärung von Gewalt sein - so wie es das Modell der soziologischen Erklärung vorsieht.

Danksagung Wir danken den anonymen Gutacher:innen und insbesondere der Redaktion des BJS für instruktive Hinweise zu einer früheren Fassung des Manuskriptes.

Funding Open Access funding enabled and organized by Projekt DEAL.

Open Access Dieser Artikel wird unter der Creative Commons Namensnennung 4.0 International Lizenz veröffentlicht, welche die Nutzung, Vervielfältigung, Bearbeitung, Verbreitung und Wiedergabe in jeglichem Medium und Format erlaubt, sofern Sie den/die ursprünglichen Autor(en) und die Quelle ordnungsgemäß nennen, einen Link zur Creative Commons Lizenz beifügen und angeben, ob Änderungen vorgenommen wurden.

Die in diesem Artikel enthaltenen Bilder und sonstiges Drittmaterial unterliegen ebenfalls der genannten Creative Commons Lizenz, sofern sich aus der Abbildungslegende nichts anderes ergibt. Sofern das betreffende Material nicht unter der genannten Creative Commons Lizenz steht und die betreffende Handlung nicht nach gesetzlichen Vorschriften erlaubt ist, ist für die oben aufgeführten Weiterverwendungen des Materials die Einwilligung des jeweiligen Rechteinhabers einzuholen.

Weitere Details zur Lizenz entnehmen Sie bitte der Lizenzinformation auf http://creativecommons.org/ licenses/by/4.0/deed.de.

\section{Literatur}

Alexander, J. C., Giesen, B., Münch, R., \& Smelser, N. (Hrsg.). (1987). The micro-macro link. Berkeley, Los Angeles: University of California Press.

Axelrod, R. (2000). Die Evolution der Kooperation. München: Oldenburg.

Bauer, F. et al. (2012). Zwischen Aktivierungsanspruch und Beschäftigungsförderung. Die Zerrissenheit gegenwärtiger Sozialpolitik im Spiegel variierender Strategien der Umsetzung von § 16e SGB II (JobPerspektive). In H.-G. Soeffner (Hrsg.), Transnationale Vergesellschaftungen. Verhandlungen des 35. Kongresses der Deutschen Gesellschaft für Soziologie in Frankfurt am Main. CD-ROM. Wiesbaden: Springer VS.

Bauman, Z. (1989). Modernity and the Holocaust. Ithacam: Cornell University Press.

Bauman, Z. (1991). Modernity and ambivalence. Ithaca: Cornell University Press.

BMFSFJ (2004). Lebenssituation, Sicherheit und Gesundheit von Frauen in Deutschland. Eine repräsentative Untersuchung zu Gewalt gegen Frauen in Deutschland. Berlin: Bundesministerium für Familie, Senioren, Frauen und Jugend.

Braun, A. (2020). Gewaltsoziologie im Wandel. In A. Braun \& T. Kron (Hrsg.), Bestandsaufnahme soziologischer Gewaltforschung. Österreichische Zeitschrift für Soziologie, Sonderheft 20 (S. 5-21). Wiesbaden: Springer VS.

Braun, A., \& Kron, T. (Hrsg.). (2020). Bestandsaufnahme soziologischer Gewaltforschung. Österreichische Zeitschrift für Soziologie, Sonderheft 20. Wiesbaden: Springer VS.

Brücher, G. (2011). Gewaltspiralen. Zur Theorie der Eskalation. Wiesbaden: VS Verlag für Sozialwissenschaften.

Coleman, J. (1991). Grundlagen der Sozialtheorie. München: Oldenbourg.

Collins, R. (2004). Interactional ritual chains. Princeton: Princeton University Press.

Collins, R. (2011). Dynamik der Gewalt. Eine mikrosoziologische Theorie. Hamburg: Hamburger Edition.

Collins, R. (2012). Entering and leaving the tunnel of violence: Micro-sociological dynamics of emotional entrainment in violent interactions. Current Sociology, 61, 132-151.

Collins, R. (2015). Vorwärtspaniken und die Dynamik von Massengewalt. In A. T. Paul \& B. Schwalb (Hrsg.), Gewaltmassen. Über Eigendynamik und Selbstorganisation kollektiver Gewalt (S. 204-230). Hamburg: Hamburger Edition. 
Collins, R. (2016). Einfahrten und Ausfahrten des Tunnels der Gewalt. In C. Equit, A. Groenmeyer \& H. Schmidt (Hrsg.), Situationen der Gewalt (S. 14-39). Weinheim: Beltz Juventa.

Collins, R. (2017). Two violent trajectories on the micro-macro-continuum: Emotional tipping-point conficts and dispersed attrition conficts. International Journal of Conflict and Violence, 11, 1-7.

Collins, R., \& Hartmann, E. (2019). An der Schwelle zur Gewalt - Ein Gespräch mit Randall Collins. Mittelweg 36, 18(1-2), 60-69.

Endreß, M. (2004). Entgrenzung des Menschlichen. Zur Transformation der Strukturen menschlichen Weltbezugs durch Gewalt. In W. Heitmeyer \& H.-G. Soeffner (Hrsg.), Gewalt (S. 174-201). Frankfurt a.M.: Suhrkamp.

Endreß, M. (2014). Grundlagenprobleme einer Soziologie der Gewalt. Zur vermeintlichen Alternative von körperlicher und struktureller Gewalt. In M. Staudigl (Hrsg.), Gesichter der Gewalt. Beiträge aus phänomenologischer Sicht (S. 87-113). München: Fink.

Equit, C., Groenmeyer, A., \& Schmidt, H. (Hrsg.). (2016). Situationen der Gewalt. Weinheim: Beltz Juventa.

Esser, H. (1993). Soziologie. Allgemeine Grundlagen. Frankfurt a.M.: Campus.

Esser, H. (1999). Soziologie. Spezielle Grundlagen. Band 1: Situationslogik und Handeln. Frankfurt a.M.: Campus.

Esser, H. (2000a). Normen als Frames: Das Problem der „Unbedingtheit“ des normativen Handelns. In R. Metze, K. Mühler \& K.-D. Opp (Hrsg.), Normen und Institutionen. Entstehung und Wirkungen (S. 137-155). Leipzig: Leipziger Universitätsverlag.

Esser, H. (2000b). Soziologie. Spezielle Grundlagen. Band 2: Die Konstruktion der Gesellschaft. Frankfurt a.M.: Campus.

Esser, H. (2001). Soziologie. Spezielle Grundlagen. Band 6: Sinn und Kultur. Frankfurt a.M.: Campus.

Esser, H. (2003a). Die Rationalität der Werte. Die Typen des Handelns und das Modell der soziologischen Erklärung. In G. Albert, A. Bienfait, S. Sigmund \& C. Wendt (Hrsg.), Das Weber-Paradigma (S. 154-188). Tübingen: Mohr.

Esser, H. (2003b). Das Modell der soziologischen Erklärung und die Paradigmen der Soziologie. In B. Orth, T. Schwietring \& J. Weiß (Hrsg.), Soziologische Forschung: Stand und Perspektiven (S. 523-532). Opladen: Leske + Budrich.

Esser, H. (2006). Affektuelles Handeln: Emotionen und das Modell der Frame-Selektion. In R. Schützeichel (Hrsg.), Emotionen und Sozialtheorie (S. 143-174). Frankfurt a.M.: Campus.

Esser, H. (2010). Sinn, Kultur, Verstehen und das Modell der soziologischen Erklärung. In M. WohlrabSahr (Hrsg.), Kultursoziologie (S. 309-355). Wiesbaden: VS Verlag für Sozialwissenschaften.

Fuchs, P. (1999). Liebe, Sex und solche Sachen. Zur Konstruktion moderner Intimsysteme. Konstanz: UVK.

Galtung, J. (1975). Strukturelle Gewalt. Beiträge zur Friedens- und Konfliktforschung. Reinbek: Rowohlt.

Gigerenzer, G. (2007). Bauchentscheidungen. Die Intelligenz des Unbewussten und die Macht der Intuition. München: Goldmann.

Gigerenzer, G., \& Todd, P. M. (1999). Simple heuristics that make us smart. New York: Oxford University Press.

Greshoff, R., Kneer, G., \& Schimank, U. (Hrsg.). (2003). Die Transintentionalität des Sozialen. Opladen: Westdeutscher Verlag.

Greve, J., Schnabel, A., \& Schützeichel, R. (Hrsg.). (2009). Das Mikro-Makro-Modell der soziologischen Erklärung. Wiesbaden: VS Verlag für Sozialwissenschaften.

Hartmann, E. (2013). Soziale Ordnung und Gewalt. Anmerkungen zur neueren Literatur in der Gewaltforschung. Berliner Journal für Soziologie, 23, 115-131.

Hartmann, E., \& Hoebel, T. (2020). Die Schweigsamkeit der Gewalt durchbrechen. WestEnd. Neue Zeitschrift für Sozialforschung, 17(1), 71-79.

Hedström, P. (2005). Dissecting the social: On the principles of analytical sociology. Cambridge: Cambridge University Press.

Hedström, P., \& Swedberg, R. (1998a). Social mechanisms: An introductory essay. In P. Hedström \& R. Swedberg (Hrsg.), Social mechanisms: An analytical approach to social theory (S. 1-31). Cambridge: Cambridge University Press.

Hedström, P., \& Swedberg, R. (Hrsg.). (1998b). Social mechanisms: An analytical approach to social theory. Cambridge: Cambridge University Press.

Hoebel, T. (2014). Organisierte Plötzlichkeit. Eine prozesssoziologische Erklärung antisymmetrischer Gewaltsituationen. Zeitschrift für Soziologie, 43, 441-457.

Hoebel, T. (2019a). Verkettungen und Verstrickungen. Skizze einer prozessualen Erklärung fortgesetzter Gewalt. Zeitschrift für Theoretische Soziologie, 8(1), 50-62. 
Hoebel, T. (2019b). „Wir haben Charlie Hebdo getötet!“ Konsequenzielle Dritte und die Erklärung fortgesetzter Gewalt. Mittelweg 36, 18(1-2), 99-123.

Hoebel, T., \& Knöbl, W. (2019). Gewalt erklären! Plädoyer für eine entdeckende Prozesssoziologie. Hamburg: Hamburger Edition.

Hoebel, T., \& Koloma Beck, T. (2017). Gewalt und ihre Indexikalität. Theoretische Potenziale einer kontextsensiblen Heuristik. ORDEX Working Paper, Nr. 4. Bielefeld: Universität Bielefeld.

Hoebel, T., \& Malthaner, S. (2019). Über dem Zenit. Grenzen und Perspektiven der situationistischen Gewaltforschung. Mittelweg 36, 18(1-2), 3-14.

Hüttermann, J. (2004). „Dichte Beschreibung“ oder Ursachenforschung der Gewalt? Anmerkungen zu einer falschen Alternative im Lichte der Problematik funktionaler Erklärungen. In W. Heitmeyer \& H.-G. Soeffner (Hrsg.), Gewalt (S. 107-124). Frankfurt a.M.: Suhrkamp.

Imbusch, P. (2004). „Mainstreamer“ versus „Innovateure“ der Gewaltforschung: Eine kuriose Debatte. In W. Heitmeyer \& H.-G. Soeffner (Hrsg.), Gewalt (S. 125-148). Frankfurt a.M.: Suhrkamp.

Imbusch, P. (2017). Strukturelle Gewalt. Plädoyer für einen unterschätzten Begriff. Mittelweg 36, 16(3), 28-51.

Knöbl, W. (2017). Perspektiven der Gewaltforschung. Mittelweg 36, 16(3), 4-27.

Koepp, T., \& Schattka, C. (2020). Eine kuriose Debatte wiederholt sich. Plädoyer für eine kontextsensible Mikrosoziologie der Gewalt. In A. Braun \& T. Kron (Hrsg.), Bestandsaufnahme soziologischer Gewaltforschung. Österreichische Zeitschrift für Soziologie, Sonderheft 20 (S. 23-42). Wiesbaden: Springer VS.

Koloma Beck, T. (2017). Gewalt als leibliche Erfahrung. Mittelweg 36, 16(3), 52-73.

Kron, T. (2006). Zur sozionischen Notwendigkeit mechanistisch-soziologischer Erklärungen. In T. Malsch \& M. Schmitt (Hrsg.), Reflexive soziale Mechanismen. Von soziologischen Erklärungen zu sozionischen Modellen (S. 105-137). Wiesbaden: VS Verlag für Sozialwissenschaften.

Kron, T. (Hrsg.). (2015). Hybride Sozialität - Soziale Hybridität. Weilerswist: Velbrück.

Kron, T. (2018). Stress und Komplexität. Zwei Grenzen des Rational-Choice-Modells. In D. Baron, O. A. Becker \& D. Lois (Hrsg.), Erklärende Soziologie und soziale Praxis (S. 15-42). Wiesbaden: Springer VS.

Kron, T. (2020a). Gewalt und emotionale Energie. In A. Braun \& T. Kron (Hrsg.), Bestandsaufnahme soziologischer Gewaltforschung. Österreichische Zeitschrift für Soziologie, Sonderheft 20 (S. 113-134). Wiesbaden: Springer VS.

Kron, T. (2020b). Moral und Gewalt - Wie viel Moral ist in Gewaltsituationen möglich? Ein Blick aus der Perspektive Zygmunt Baumans. In K. Platt (Hrsg.), Fehlfarben der Postmoderne. Weiter-Denken mit Zygmunt Bauman (S. 304-323). Weilerswist: Velbrück.

Kron, T., \& Grund, T. (2009). Society as a self-organized critical system. Cybernetics \& Human Knowing, $19(1-2), 65-82$.

Lessenich, S. (2016). Neben uns die Sintflut. Die Externalisierungsgesellschaft und ihr Preis. Berlin: Hanser.

Lester, D., Wood, P., Williams, C., \& Haines, J. (2004). Motives for suicide - A study of Australian suicide notes. Crisis, 25, 33-34.

Lindemann, G. (2015). Gewalt als soziologische Kategorie. Archiv für Rechts- und Sozialphilosophie, 101, $501-512$.

Lindemann, G. (2017). Verfahrensordnungen der Gewalt. Zeitschrift für Rechtssoziologie, 37, 57-87.

Mahoney, J. (2000). Path dependence in historical sociology. Theory and Society, 29, 507-548.

Marneros, A. (2008). Intimizid - Die Tötung des Intimpartners. Stuttgart: Schattenauer.

Meyer, P. (2004). Grundlagen menschlicher Gewaltbereitschaft. Beiträge evolutionärer Forschung. In W. Heitmeyer \& H.-G. Soeffner (Hrsg.), Gewalt (S. 383-410). Frankfurt a.M.: Suhrkamp.

Miller, R. (2011). Facing violence. Preparing for the unexpected. Wolfeboro: YMAA.

Münch, R. (1986). Die Kultur der Moderne. Frankfurt a.M.: Suhrkamp.

Münch, R. (1998). Rational Choice. Grenzen der Erklärungskraft. In H.-P. Müller \& M. Schmid (Hrsg.), Norm, Herrschaft und Vertrauen. Beiträge zu James S. Colemans Grundlagen der Sozialtheorie (S. 79-91). Opladen: Westdeutscher Verlag.

Nassauer, A. (2012). Violence in demonstrations: A comparative analysis of situational interaction dynamics at social movement protests. Dissertation. Berlin: Humboldt-Universität zu Berlin.

Nassauer, A. (2015a). Effective crowd policing: Empirical insights on avoiding violence. Policing: An International Journal of Police Strategies \& Management, 38, 3-23.

Nassauer, A. (2015b). Developing a micro-situational theory of crowd violence. In E. C. Kebler (Hrsg.), Cultures, social bonds and the dynamics of violence (S. 39-53). Oxfordshire: Inter-Disciplinary Press. 
Nassauer, A. (2015c). Theoretische Überlegungen zur Entstehung von Gewalt in Protesten: Eine situative mechanismische Erklärung. Berliner Journal für Soziologie, 25, 491-518.

Nassauer, A. (2016). From peaceful marches to violent clashes: A micro-situational analysis. Social Movement Studies, 15(5), 1-16.

Nedelmann, B. (1997). Gewaltsoziologie am Scheideweg. Die Auseinandersetzungen in der gegenwärtigen und Wege der künftigen Gewaltforschung. In T. von Trotha (Hrsg.), Soziologie der Gewalt (S. 59-85). Opladen: Westdeutscher Verlag.

Nungesser, F. (2017). Ein pleonastisches Oxymoron. Konstruktionsprobleme von Pierre Bourdieus Schlüsselkonzept der symbolischen Gewalt. Berliner Journal für Soziologie, 27, 7-33.

Nungesser, F. (2019). Die Vielfalt der Verletzbarkeit und die Ambivalenz der Sensibilität. Zeitschrift für Theoretische Soziologie, 8(1), 24-37.

Nunner-Winkler, G. (2004). Überlegungen zum Gewaltbegriff. In W. Heitmeyer \& H.-G. Soeffner (Hrsg.), Gewalt (S. 21-61). Frankfurt a.M.: Suhrkamp.

Paul, A. T., \& Schwalb, B. (Hrsg.). (2015). Gewaltmassen. Über Eigendynamik und Selbstorganisation kollektiver Gewalt. Hamburg: Hamburger Edition.

Popitz, H. (1992). Phänomene der Macht. 2., stark erw. Aufl. Tübingen: Mohr.

Preiser, C. (2016). Gewalt im Arbeitsalltag von Türstehern. In C. Equit, A. Groenmeyer \& H. Schmidt (Hrsg.), Situationen der Gewalt (S. 323-341). Weinheim: Beltz Juventa.

Ragin, C. C. (1986). The comparative method: Moving beyond qualitative and quantitative strategies. Berkeley: University of California Press.

Ragin, C. C. (2000). Fuzzy-set social science. Chicago: University of Chicago Press.

Reemtsma, J. P. (2017). Erklärungsbegehren. Mittelweg 36, 16(3), 74-103.

Riekenberg, M. (2008). Auf dem Holzweg? Über Johan Galtungs Begriff der „strukturellen Gewalt“. Zeithistorische Forschungen, 5, 172-177.

Rotte, R. (2019). Das Phänomen Krieg. Eine sozialwissenschaftliche Bestandsaufnahme. Wiesbaden: Springer VS.

Schimank, U. (1985). Der mangelnde Akteurbezug systemtheoretischer Erklärungen gesellschaftlicher Differenzierung - Ein Diskussionsvorschlag. Zeitschrift für Soziologie, 14, 421-434.

Schimank, U. (2000). Handeln und Strukturen. Weinheim: Juventus.

Schimank, U. (2005). Die Entscheidungsgesellschaft. Komplexität und Rationalität der Moderne. Wiesbaden: VS Verlag für Sozialwissenschaften.

Schmid, M. (2006). Die Logik mechanismischer Erklärungen. Wiesbaden: VS Verlag für Sozialwissenschaften.

Schroer, M. (2004). Gewalt ohne Gesicht. Zur Notwendigkeit einer umfassenden Gewaltanalyse. In W. Heitmeyer \& H.-G. Soeffner (Hrsg.), Gewalt (S. 151-173). Frankfurt a.M.: Suhrkamp.

Simmel, G. (1922). Lebensanschauung. Vier metaphysische Kapitel. München: Duncker \& Humblot.

Sofsky, W. (1996). Traktat über die Gewalt. Frankfurt a.M.: Fischer.

Staack, M. (2015). Praktiken der Gewalt? Randall Collins' Konzept der Confrontational Tension/Fear in der Analyse des Kampfsporttrainings. In S. Liebl \& P. Kuhn (Hrsg.), Menschen im Zweikampf (S. 176-184). Hamburg: Feldhaus.

Staudigl, M. (2014). (Hrsg.). Gesichter der Gewalt. Beiträge aus phänomenologischer Sicht. München: Fink.

Staudigl, M. (2015). Phänomenologie der Gewalt. Springer: Cham.

Sutterlüty, F. (2015). Kollektive Gewalt und urbane Riots. Was erklärt die Situation? In A. Paul \& B. Schwalb (Hrsg.), Gewaltmassen. Über Eigendynamik und Selbstorganisation kollektiver Gewalt (S. 231-256). Hamburg: Hamburger Edition.

Sutterlüty, F. (2020). Das strukturelle Gewaltpotential der Familie. WestEnd. Neue Zeitschrift für Sozialforschung, 17(1), 93-102.

Swaine, J., Laughland, O., Lartaey, J., \& McCarthy, C. (2015). Young black men killed by US police at highest rate in year of 1,134 deaths. The Guardian vom 31.12.2015. https://www.theguardian.com/ us-news/2015/dec/31/the-counted-police-killings-2015-young-black-men. Zugegriffen: Jan. 2018.

Thornhill, R., \& Palmer, C. T. (2000). A natural history of rape. Biological bases of sexual coercion. Cambridge: MIT Press.

von Trotha, Trutz (1997). Zur Soziologie der Gewalt. In T. von Trotha (Hrsg.), Soziologie der Gewalt (S. 9-56). Opladen: Westdeutscher Verlag.

Unterkoffler, U. (2016). Wo beginnt Gewalt? In C. Equit, A. Groenmeyer \& H. Schmidt (Hrsg.), Situationen der Gewalt (S. 120-149). Weinheim: Beltz Juventa.

Weber, M. (1980). Wirtschaft und Gesellschaft. Grundriß der verstehenden Soziologie. 5., rev. Aufl. Tübingen: Mohr. 
Wolters, L. (2020). Gewaltforschung im Modus des Erklärens. WestEnd. Neue Zeitschrift für Sozialforschung, 17(1), 115-124.

Thomas Kron geb. 1970. Dr. rer. pol., Universitätsprofessor für Soziologie an der RWTH Aachen. Aktuelle Forschungsschwerpunkte: Soziologische Theorie, insbesondere Handlungs- und Systemtheorien sowie zur Integration der Fuzzy-Logik in die Soziologie, Gewaltforschung. Ausgewählte aktuelle Veröffentlichungen: (mit A. Braun, Hrsg.) Bestandsaufnahme soziologischer Gewaltforschung. Sonderheft der Österreichischen Zeitschrift für Soziologie, 2020; (mit C. Laut) Bystander im Clear-Net - Der Fall Heße, in: Zeitschrift für Soziologie, 2019; (mit L. Winter) Die (Re)Produktion des Terrors - Unterscheidungen und Vagheiten, in: Soziale Systeme, 2018.

Lena M. Verneuer geb. 1986. Dr. phil., wissenschaftliche Mitarbeiterin am Institut für Soziologie der RWTH Aachen. Forschungsschwerpunkte: Soziologie der Kriminalität und Gewalt, Methoden der empirischen Sozialforschung, Handlungstheorien. Ausgewählte Veröffentlichungen: Gewalt als Sanktionshandlung. Eine kriminalsoziologische Analyse physischer Gewalthandlungen, 2020; Selbstbericht und Vignette als Instrumente zur empirischen Abbildung von Gewalt als Sanktionshandlung, in: I. Krumpal und R. Berger (Hrsg.), Devianz und Subkulturen. Theorien, Methoden und empirische Befunde, 2020. 